\title{
HESSIAN VALUATIONS
}

\author{
ANDREA COLESANTI, MONIKA LUDWIG \& FABIAN MUSSNIG
}

\begin{abstract}
A new class of continuous valuations on the space of convex functions on $\mathbb{R}^{n}$ is introduced. On smooth convex functions, they are defined for $i=0, \ldots, n$ by

$$
u \mapsto \int_{\mathbb{R}^{n}} \zeta(u(x), x, \nabla u(x))\left[\mathrm{D}^{2} u(x)\right]_{i} \mathrm{~d} x
$$

where $\zeta \in C\left(\mathbb{R} \times \mathbb{R}^{n} \times \mathbb{R}^{n}\right)$ and $\left[\mathrm{D}^{2} u\right]_{i}$ is the $i$ th elementary symmetric function of the eigenvalues of the Hessian matrix, $\mathrm{D}^{2} u$, of $u$. Under suitable assumptions on $\zeta$, these valuations are shown to be invariant under translations and rotations on convex and coercive functions.
\end{abstract}

2000 AMS subject classification: 52B45 (26B25, 49Q20, 52A21, 52A41)

\section{INTRODUCTION}

The purpose of this paper is to introduce a new type of valuations on convex functions. On smooth convex functions, they are defined for $i=0, \ldots, n$ by

$$
u \mapsto \int_{\mathbb{R}^{n}} \zeta(u(x), x, \nabla u(x))\left[\mathrm{D}^{2} u(x)\right]_{i} \mathrm{~d} x,
$$

where $\zeta: \mathbb{R} \times \mathbb{R}^{n} \times \mathbb{R}^{n} \rightarrow \mathbb{R}$ is continuous and $\left[\mathrm{D}^{2} u\right]_{i}$ is the $i$ th elementary symmetric function of the eigenvalues of the Hessian matrix of $u$, with the usual convention $\left[\mathrm{D}^{2} u\right]_{0}=1$ (alternatively, $\left[\mathrm{D}^{2} u\right]_{i}$ is the sum of the $i \times i$ principal minors of $\mathrm{D}^{2} u$ ). We show that these functionals can be extended to a rather ample class of convex functions, providing a family of continuous valuations that we call Hessian valuations. Here continuity is with respect to epi-convergence (see Section 2). Under suitable assumptions on $\zeta$, Hessian valuations are invariant under rotations on convex functions and under translations and rotations on convex and coercive functions.

The theory of valuations on function spaces has been rapidly growing in recent years. Several spaces of functions have been investigated already, including Lebesgue and Sobolev spaces, functions of bounded variations, and quasi-concave functions (see [3-5, 9, 12, 13, 22- -27, 31, 37- 39]). Needless to say, the major impulse to this area comes from the rich and beautiful theory of valuations defined on the family $\mathcal{K}^{n}$ of convex bodies (that is, compact convex subsets) in $\mathbb{R}^{n}$, which is one of the most active branches of convex geometry (see [35, Chapter 6] for a recent survey on the theory of valuations on convex bodies).

As a general framework, we start from the following family of convex functions:

$$
\operatorname{Conv}\left(\mathbb{R}^{n}\right)=\left\{u: \mathbb{R}^{n} \rightarrow \mathbb{R} \cup\{+\infty\}: u \text { convex and 1.s.c., } u \not \equiv+\infty\right\},
$$

where 1.s.c. stands for lower semicontinuous. A (real-valued) valuation on $\operatorname{Conv}\left(\mathbb{R}^{n}\right)$ is a functional $\mathrm{Z}: \operatorname{Conv}\left(\mathbb{R}^{n}\right) \rightarrow \mathbb{R}$ which has the additivity property

$$
\mathrm{Z}(u \vee v)+\mathrm{Z}(u \wedge v)=\mathrm{Z}(u)+\mathrm{Z}(v),
$$

for every $u, v \in \operatorname{Conv}\left(\mathbb{R}^{n}\right)$ such that $u \wedge v \in \operatorname{Conv}\left(\mathbb{R}^{n}\right)$. Here $\vee$ and $\wedge$ denote the pointwise maximum and minimum, respectively. 
The basic tool to extend a functional of the form (1.1) to $\operatorname{Conv}\left(\mathbb{R}^{n}\right)$ are Hessian measures of convex functions (see Section 7), that will be denoted by $\Theta_{i}(u, \cdot)$ for $i \in\{0, \ldots, n\}$ and $u \in \operatorname{Conv}\left(\mathbb{R}^{n}\right)$. Like support measures of convex bodies, Hessian measures can be defined as coefficients of a local Steiner formula,

$$
\mathcal{H}^{n}\left(P_{s}(u, \eta)\right)=\sum_{i=0}^{n}\left(\begin{array}{c}
n \\
i
\end{array}\right) s^{i} \Theta_{n-i}(u, \eta),
$$

where $\mathcal{H}^{n}$ is the $n$-dimensional Hausdorff measure and $s \geq 0$ while for a Borel subset $\eta$ of $\mathbb{R}^{n} \times \mathbb{R}^{n}$,

$$
P_{s}(u, \eta)=\{x+s y:(x, y) \in \eta, y \in \partial u(x)\} .
$$

Here $\partial u(x)$ denotes the subdifferential of $u$ at the point $x$ (see Section2). Hessian measures permit to extend to non-smooth convex functions integrals of the elementary symmetric functions of the eigenvalues of the Hessian matrix. Indeed, if $u \in \operatorname{Conv}\left(\mathbb{R}^{n}\right) \cap C^{2}\left(\mathbb{R}^{n}\right)$ and $i \in\{0, \ldots, n\}$, then

$$
\Theta_{i}\left(u, \beta \times \mathbb{R}^{n}\right)=\int_{\beta}\left[\mathrm{D}^{2} u(x)\right]_{n-i} \mathrm{~d} x
$$

for every Borel subset $\beta$ of $\mathbb{R}^{n}$. Hessian measures have been considered in connection with convex (and more general) functions in $[8,10,11,18]$ and are related to non-linear elliptic partial differential equations (see, e.g., [6, 16, 36]).

To define the integral (1.1) for an arbitrary $u \in \operatorname{Conv}\left(\mathbb{R}^{n}\right)$, we integrate $\zeta(u(x), x, y)$ with respect to the Hessian measure of $u$, where the variable $y$ plays the role of $\nabla u$. To guarantee integrability, we assume that $\zeta(t, x, y)$ has compact support with respect to the second and third variables, that is, there exists $r>0$ such that $\zeta$ vanishes in the complement set of the cylinder $\{(t, x, y):|x| \leq r,|y| \leq r\}$, where $|\cdot|$ is the Euclidean norm. Our first result is the following.

Theorem 1.1. Let $\zeta \in C\left(\mathbb{R} \times \mathbb{R}^{n} \times \mathbb{R}^{n}\right)$ have compact support with respect to the second and third variables. For every $i \in\{0,1, \ldots, n\}$, the functional $\mathrm{Z}_{\zeta, i}: \operatorname{Conv}\left(\mathbb{R}^{n}\right) \rightarrow \mathbb{R}$, defined by

$$
\mathrm{Z}_{\zeta, i}(u)=\int_{\mathbb{R}^{2 n}} \zeta(u(x), x, y) \mathrm{d} \Theta_{i}(u,(x, y)),
$$

is a continuous valuation on $\operatorname{Conv}\left(\mathbb{R}^{n}\right)$. If $u \in \operatorname{Conv}\left(\mathbb{R}^{n}\right) \cap C^{2}\left(\mathbb{R}^{n}\right)$, then $\mathrm{Z}_{\zeta, i}(u)$ takes the form (1.1).

For $\zeta$ and $i$ given as above, two additional properties of $\mathrm{Z}_{\zeta, i}$ will be established.

(i) The functional $\mathrm{Z}_{\zeta, i}$ is $i$-simple, that is, we have $\mathrm{Z}_{\zeta, i}(u)=0$ for all $u \in \operatorname{Conv}\left(\mathbb{R}^{n}\right)$ such that $\operatorname{dim}(\operatorname{dom}(u))<i$, where $\operatorname{dom}(u)=\left\{x \in \mathbb{R}^{n}: u(x)<+\infty\right\}$ is the domain of $u$ and dim stands for dimension.

(ii) If $\zeta$ is of the form $\zeta(t, x, y)=\xi(t,|x|,|y|)$ for some $\xi \in C\left(\mathbb{R} \times[0,+\infty)^{2}\right)$, then (1.3) is invariant under rotations, that is, $\mathrm{Z}_{\zeta, i}(u)=\mathrm{Z}_{\zeta, i}\left(u \circ \phi^{-1}\right)$ for every $\phi \in \mathrm{O}(n)$.

Remark. In [3], Alesker considers the space of convex functions on an open and convex set $U \subset \mathbb{R}^{n}$. Using Monge-Ampère measures, he introduces a class of valuations which extend to general convex functions functionals of the form

$$
u \mapsto \int_{U} \xi(x) \operatorname{det}\left(\mathrm{D}^{2} u(x), \ldots, \mathrm{D}^{2} u(x), A_{k+1}(x), \ldots, A_{n}(x)\right) \mathrm{d} x,
$$

where the function $\xi \in C\left(\mathbb{R}^{n}\right)$ has compact support, det is the mixed discriminant operator of $n$ matrices, the Hessian $\mathrm{D}^{2} u(x)$ appears $k$ times with $k \in\{0, \ldots, n\}$, and $A_{i}$ is a symmetric $n \times n$ matrix having as coefficients continuous and compactly supported functions for every $i \in\{k+1, \ldots, n\}$. There is an overlap between Alesker's valuations and the ones introduced here. Indeed, for $U=\mathbb{R}^{n}$, if $\zeta=\xi$ 
depends on the $x$-variable only and we choose $A_{i} \equiv I_{n}$ for $i=k+1, \ldots, n$, where $I_{n}$ is the identity matrix of order $n$, then the notions coincide for every $u \in \operatorname{Conv}\left(\mathbb{R}^{n}\right) \cap C^{2}\left(\mathbb{R}^{n}\right)$.

In [7, 14, 15, 30], valuations defined on the space of convex and coercive functions,

$$
\operatorname{Conv}_{\text {coe }}\left(\mathbb{R}^{n}\right)=\left\{u: \mathbb{R}^{n} \rightarrow \mathbb{R} \cup\{+\infty\}: u \text { convex, 1.s.c., and coercive, } u \not \equiv+\infty\right\}
$$

were studied and classified. Here a function $u: \mathbb{R}^{n} \rightarrow \mathbb{R} \cup\{+\infty\}$ is coercive if $\lim _{|x| \rightarrow+\infty} u(x)=+\infty$. In [7, 15], the following type of valuations on $\operatorname{Conv}_{\text {coe }}\left(\mathbb{R}^{n}\right)$ were considered for suitable $\zeta: \mathbb{R} \rightarrow \mathbb{R}$ :

$$
u \mapsto \int_{\mathbb{R}^{n}} \zeta(u(x)) \mathrm{d} x
$$

In [15], it is proved that (1.4) defines a continuous and rigid motion invariant valuation on $\operatorname{Conv}_{\text {coe }}\left(\mathbb{R}^{n}\right)$ if and only if $\zeta$ is continuous on $\mathbb{R}$ and has finite $(n-1)$ th moment. Moreover, it is proved that every continuous, non-negative, $\mathrm{SL}(n)$ and translation invariant valuation on $\operatorname{Conv}_{\text {coe }}\left(\mathbb{R}^{n}\right)$ can be written as a functional of the form (1.4) plus a function only depending on $\min _{\mathbb{R}^{n}} u$.

We obtain new valuations on $\operatorname{Conv}_{\text {coe }}\left(\mathbb{R}^{n}\right)$. The coercivity guarantees that integrability is preserved under less restrictive conditions on $\zeta$. In particular, we may remove the dependence on the space variable $x$, gaining translation invariance.

Theorem 1.2. Let $\zeta \in C\left(\mathbb{R} \times \mathbb{R}^{n}\right)$ have compact support. For every $i \in\{0, \ldots, n\}$, the functional $\mathrm{Z}_{\zeta, i}: \operatorname{Conv}_{\text {coe }}\left(\mathbb{R}^{n}\right) \rightarrow \mathbb{R}$, defined by

$$
\mathrm{Z}_{\zeta, i}(u)=\int_{\mathbb{R}^{2 n}} \zeta(u(x), y) \mathrm{d} \Theta_{i}(u,(x, y)),
$$

is a continuous, translation invariant, $i$-simple valuation. Moreover, if there exists $\xi \in C(\mathbb{R} \times[0,+\infty))$ such that $\zeta(t, y)=\xi(t,|y|)$ on $\mathbb{R} \times \mathbb{R}^{n}$, then $\mathrm{Z}_{\zeta, i}$ is also rotation invariant.

Hence, for every $\zeta \in C(\mathbb{R} \times[0,+\infty))$ with compact support, the valuation

$$
u \mapsto \int_{\mathbb{R}^{2 n}} \zeta(u(x),|y|) \mathrm{d} \Theta_{i}(u,(x, y))
$$

is continuous and rigid motion invariant for $i \in\{0, \ldots, n\}$.

We mention that in [7] the construction of a class of valuations on $\operatorname{Conv}_{\text {coe }}\left(\mathbb{R}^{n}\right)$ is presented, based on quermassintegrals (or intrinsic volumes) of level sets. Similar ideas can be found in [5, 28, 29]. A comparison between those and Hessian valuations is carried out in Section 11.1, where in particular we show that there are Hessian valuations that are not included in the class described in [7].

In the following, we collect results needed for the preparation of the proofs of the main results. In Section 3, we recall some basic facts on convex functions. In Section 4, the Lipschitz regularization of convex functions is described. Sections 5 and 6 are devoted to inclusion-exclusion type properties of the subdifferential, the subdifferential graph and local parallel sets of convex functions. These properties will be critical to prove the valuation property of Hessian measures, which is discussed in Sections 7 and 8. In particular, in Section 7 we extend to $\operatorname{Conv}\left(\mathbb{R}^{n}\right)$ the results of [11] concerning existence and integral representations of Hessian measures, while in the next section we discuss several (known and new) facts about them.

Subsequently, after giving the main definitions for valuations on $\operatorname{Conv}\left(\mathbb{R}^{n}\right)$ in Section 9, we prove Theorems 1.1 and 1.2 in Sections 10 and 11. In Section 12, we briefly analyze what happens if we restrict Hessian valuations to the subset $\operatorname{Conv}_{\text {hom }}\left(\mathbb{R}^{n}\right)$ of $\operatorname{Conv}\left(\mathbb{R}^{n}\right)$, formed by finite convex functions homogeneous of degree 1 , that is, by support functions of convex bodies. Note that continuous valuations on $\operatorname{Conv}_{\text {hom }}\left(\mathbb{R}^{n}\right)$ correspond to continuous valuations on $\mathcal{K}^{n}$. 


\section{PRELIMINARIES}

We work in $n$-dimensional Euclidean space, $\mathbb{R}^{n}$, for $n \geq 1$, endowed with the usual Euclidean norm $|\cdot|$ and scalar product $\langle\cdot, \cdot\rangle$. We set $\mathbb{S}^{n-1}=\left\{x \in \mathbb{R}^{n}:|x|=1\right\}$ and $B^{n}=\left\{x \in \mathbb{R}^{n}:|x| \leq 1\right\}$. For $r>0$, let $B_{r}^{n}$ be the closed ball of $\mathbb{R}^{n}$, centered at the origin and with radius $r$. Given a subset $A$ of $\mathbb{R}^{n}$, its interior and boundary will be denoted by $\operatorname{int}(A)$ and $\operatorname{bd}(A)$, respectively. For $k \in[0, n]$, the $k$-dimensional Hausdorff measure in $\mathbb{R}^{n}$ is denoted by $\mathcal{H}^{k}$. In particular, $\mathcal{H}^{n}$ is the Lebesgue measure in $\mathbb{R}^{n}$. We write $\pi_{1}$ and $\pi_{2}$ for the canonical projections of $\mathbb{R}^{n} \times \mathbb{R}^{n}$ onto the first and the second component, respectively; that is,

$$
\pi_{1}(x, y)=x, \quad \pi_{2}(x, y)=y
$$

for $(x, y) \in \mathbb{R}^{n} \times \mathbb{R}^{n}$.

A subset $C$ of $\mathbb{R}^{n}$ is convex if for every $x_{0}, x_{1} \in C$ and $t \in[0,1]$, we have $(1-t) x_{0}+t x_{1} \in C$. If $C \subset \mathbb{R}^{n}$ is convex, we define its dimension, $\operatorname{dim}(C)$, as the minimum integer $k \in\{0,1, \ldots, n\}$ such that there exists an affine subspace of dimension $k$ containing $C$. The relative interior of a convex set $C$ of dimension $k$ is the subset of those points $x$ of $C$ for which there exists a $k$-dimensional ball centered at $x$ and contained in $C$. The relative interior will be denoted by relint $(C)$. Note that $\operatorname{dim}(C)=n$ if and only if $\operatorname{relint}(C)=\operatorname{int}(C)$. The group of rotations of $\mathbb{R}^{n}$ will be denoted by $\mathrm{O}(n)$. By a rigid motion we mean the composition of a translation and an element of $\mathrm{O}(n)$.

A convex body is a compact convex subset of $\mathbb{R}^{n}$. We will denote by $\mathcal{K}^{n}$ the family of convex bodies in $\mathbb{R}^{n}$. Our main reference for the theory of convex body is the monograph [35]. We will sometimes need separation theorems, especially for convex sets. For the notion of separation, strict separation and strong separation of two subsets of $\mathbb{R}^{n}$ by a hyperplane we refer to [35, Section 1.3].

If $u$ and $v$ are functions defined in $\mathbb{R}^{n}$, taking values in $\mathbb{R}$ or in $\mathbb{R} \cup\{+\infty\}$, we denote by $u \vee v$ and $u \wedge v$ the pointwise maximum function and the pointwise minimum function of $u$ and $v$, respectively. In other words, for $x \in \mathbb{R}^{n}$,

$$
(u \vee v)(x)=\max \{u(x), v(x)\}, \quad(u \wedge v)(x)=\min \{u(x), v(x)\} .
$$

For $u: \mathbb{R}^{n} \rightarrow \mathbb{R} \cup\{+\infty\}$, we define its epigraph by

$$
\operatorname{epi}(u)=\left\{(x, t) \in \mathbb{R}^{n} \times \mathbb{R}: t \geq u(x)\right\} .
$$

We say that a function $w: \mathbb{R}^{n} \rightarrow \mathbb{R}$ is affine if there exist $y \in \mathbb{R}^{n}$ and $c \in \mathbb{R}$ such that $w(x)=\langle x, y\rangle+c$ for $x \in \mathbb{R}^{n}$. We denote by $\operatorname{Aff}\left(\mathbb{R}^{n}\right)$ the family of affine functions on $\mathbb{R}^{n}$.

\section{Convex Functions}

A function $u: \mathbb{R}^{n} \rightarrow \mathbb{R} \cup\{+\infty\}$ is convex if for every $x_{0}, x_{1} \in \mathbb{R}^{n}$ and for every $t \in[0,1]$, we have

$$
u\left((1-t) x_{0}+t x_{1}\right) \leq(1-t) u\left(x_{0}\right)+t u\left(x_{1}\right) .
$$

Recall that a function $u$ is convex if and only if the epigraph of $u$ is a convex subset of $\mathbb{R}^{n} \times \mathbb{R}$. Note that the lower semicontinuity of $u$ is equivalent to epi $(u)$ being closed. Such functions are also called closed. Our main reference texts on convex analysis - the theory of convex functions - are [33] and [34].

For $u \in \operatorname{Conv}\left(\mathbb{R}^{n}\right)$, we define the domain of $u$ as

$$
\operatorname{dom}(u):=\left\{x \in \mathbb{R}^{n}: u(x)<+\infty\right\} .
$$

By the convexity of $u$, its domain is a convex set. Every convex function $u$ is continuous in the interior of $\operatorname{dom}(u)$. 
Example 3.1. Let $K$ be a convex body in $\mathbb{R}^{n}$. The convex indicator function, $I_{K}: \mathbb{R}^{n} \rightarrow \mathbb{R} \cup\{+\infty\}$, of $K$ is defined by

$$
I_{K}(x)= \begin{cases}0 & \text { if } x \in K, \\ +\infty & \text { if } x \notin K .\end{cases}
$$

Hence $I_{K} \in \operatorname{Conv}_{\text {coe }}\left(\mathbb{R}^{n}\right)$ for every $K \in \mathcal{K}^{n}$.

Example 3.2. Another function associated to a convex body $K$ is its support function, denoted by $h_{K}$ and defined on $\mathbb{R}^{n}$ by

$$
h_{K}(y)=\sup _{x \in K}\langle x, y\rangle
$$

(see [35, Chapter 1]). The support function $h_{K}$ is a 1-homogeneous convex function for every $K \in \mathcal{K}^{n}$, where $f: \mathbb{R}^{n} \rightarrow \mathbb{R}$ is called 1-homogeneous, if $f(t x)=t u(x)$ for all $t>0$ and $x \in \mathbb{R}^{n}$. In particular, the support function is everywhere finite by the compactness of $K$.

The pointwise maximum of two convex functions is again a convex function, but this does not guarantee that $\operatorname{Conv}\left(\mathbb{R}^{n}\right)$ is closed with respect to $\vee$, as $u \vee v$ may be identically $+\infty$ for some $u, v \in \operatorname{Conv}\left(\mathbb{R}^{n}\right)$. On the other hand, it is easy to see that if $u, v \in \operatorname{Conv}\left(\mathbb{R}^{n}\right)$ are such that $u \wedge v \in \operatorname{Conv}\left(\mathbb{R}^{n}\right)$, then $u \vee v \in \operatorname{Conv}\left(\mathbb{R}^{n}\right)$.

3.1. The conjugate of a convex function. We recall the notion of conjugate of a convex function (see [33]). For $u \in \operatorname{Conv}\left(\mathbb{R}^{n}\right)$ and $y \in \mathbb{R}^{n}$, we set

$$
u^{*}(y):=\sup _{x \in \mathbb{R}^{n}}(\langle x, y\rangle-u(x)) .
$$

As every function $u \in \operatorname{Conv}\left(\mathbb{R}^{n}\right)$ is closed with $u \not \equiv+\infty$, the following result is a consequence of Theorem 12.2 and Corollary 12.2.1 in [33].

Proposition 3.3. If $u \in \operatorname{Conv}\left(\mathbb{R}^{n}\right)$, then $u^{*} \in \operatorname{Conv}\left(\mathbb{R}^{n}\right)$ and $u^{* *}:=\left(u^{*}\right)^{*}=u$.

The next result follows easily from [33, Theorem 16.5].

Proposition 3.4. If $u, v \in \operatorname{Conv}\left(\mathbb{R}^{n}\right)$ are such that $u \wedge v \in \operatorname{Conv}\left(\mathbb{R}^{n}\right)$, then

$$
(u \wedge v)^{*}=u^{*} \vee v^{*},
$$

and

$$
(u \vee v)^{*}=u^{*} \wedge v^{*}
$$

In particular $u^{*} \wedge v^{*}$ is convex.

Proof. For every $y \in \mathbb{R}^{n}$, we have

$$
\begin{aligned}
(u \wedge v)^{*}(y) & =\sup _{x \in \mathbb{R}^{n}}(\langle x, y\rangle-(u \wedge v)(x)) \\
& =\sup _{x \in \mathbb{R}^{n}}(\max \{\langle x, y\rangle-u(x),\langle x, y\rangle-v(x)\}) \\
& =\max \left\{\sup _{x \in \mathbb{R}^{n}}\left(\langle x, y\rangle-u(x), \sup _{x \in \mathbb{R}^{n}}(\langle x, y\rangle-v(x))\right\}\right. \\
& =\left(u^{*} \vee v^{*}\right)(y) .
\end{aligned}
$$

This proves (3.1). Together with Proposition 3.3 this gives

$$
\left(u^{*} \wedge v^{*}\right)=\left(\left(u^{*} \wedge v^{*}\right)^{*}\right)^{*}=\left(\left(u^{*}\right)^{*} \vee\left(v^{*}\right)^{*}\right)^{*}=(u \vee v)^{*},
$$

which shows (3.2). 
3.2. Topology in $\operatorname{Conv}\left(\mathbb{R}^{n}\right)$. As in [15] and [14], we adopt the topology induced by epi-convergence. A sequence $u_{k}$ of elements of $\operatorname{Conv}\left(\mathbb{R}^{n}\right)$ is epi-convergent to $u \in \operatorname{Conv}\left(\mathbb{R}^{n}\right)$ if for every $x \in \mathbb{R}^{n}$ the following conditions hold.

(i) For every sequence $x_{k}$ that converges to $x$,

$$
u(x) \leq \liminf _{k \rightarrow+\infty} u_{k}\left(x_{k}\right) .
$$

(ii) There exists a sequence $x_{k}$ that converges to $x$ such that

$$
u(x)=\lim _{k \rightarrow+\infty} u_{k}\left(x_{k}\right) .
$$

An exhaustive source for epi-convergence of convex functions is [34], where also the following result can be found.

Proposition 3.5. A sequence $u_{k}$ of functions from $\operatorname{Conv}\left(\mathbb{R}^{n}\right)$ epi-converges to $u \in \operatorname{Conv}\left(\mathbb{R}^{n}\right)$ if and only if the sequence $u_{k}^{*}$ epi-converges to $u^{*}$.

Remark. If $u_{k}$ is a sequence of finite functions in $\operatorname{Conv}\left(\mathbb{R}^{n}\right)$, that is, $\operatorname{dom}\left(u_{k}\right)=\mathbb{R}^{n}$ for every $k$, and $u \in \operatorname{Conv}\left(\mathbb{R}^{n}\right)$ is also finite, then $u_{k}$ epi-converges to $u$ if and only if it converges to $u$ pointwise in $\mathbb{R}^{n}$ and uniformly on compact sets (see for instance [34, Theorem 7.17]).

3.3. Subdifferentials. Let $u \in \operatorname{Conv}\left(\mathbb{R}^{n}\right)$ and $x \in \mathbb{R}^{n}$. A vector $y \in \mathbb{R}^{n}$ is said to be a subgradient of $u$ at $x$ if

$$
u(z) \geq u(x)+\langle z-x, y\rangle
$$

for all $z \in \mathbb{R}^{n}$. The (possibly empty) set of all vectors with this property will be denoted by $\partial u(x)$ and called the subdifferential of $u$ at $x$. In particular, $\partial u(x)=\emptyset$ for every $x \notin \operatorname{dom}(u)$. Also note that if $x \in \mathbb{R}^{n}$ is such that $\partial u(x) \neq \emptyset$, then $\partial u(x)$ is closed and convex.

Remark. If $u \in \operatorname{Conv}\left(\mathbb{R}^{n}\right)$, then there exists at least one point $x \in \mathbb{R}^{n}$ such that $\partial u(x) \neq \emptyset$, since $\operatorname{dom}(u) \neq \emptyset$ implies that relint $(\operatorname{dom}(u)) \neq \emptyset$ and by [33, p. 227].

The subdifferential is strongly connected to directional derivatives. Let $u \in \operatorname{Conv}\left(\mathbb{R}^{n}\right)$. Following the notation used in [33], for $x \in \operatorname{dom}(u)$ and a vector $y \in \mathbb{R}^{n}$, we set

$$
u^{\prime}(x ; y)=\lim _{t \rightarrow 0^{+}} \frac{u(x+t y)-u(x)}{t} .
$$

This limit always exists, finite or not, that is, $u^{\prime}(x ; y) \in \mathbb{R} \cup\{ \pm \infty\}$ (see [33, Theorem 23.1]). The following result is [33, Theorem 23.2].

Lemma 3.6. Let $u \in \operatorname{Conv}\left(\mathbb{R}^{n}\right)$ and $x \in \operatorname{dom}(u)$. For $y \in \mathbb{R}^{n}$, we have $y \in \partial u(x)$ if and only if $\langle z, y\rangle \leq u^{\prime}(x ; z)$ for all $z \in \mathbb{R}^{n}$.

We also need the following result, which can be found in [33, Theorem 23.5].

Lemma 3.7. For $u \in \operatorname{Conv}\left(\mathbb{R}^{n}\right)$ and $x, y \in \mathbb{R}^{n}$, the following are equivalent:

(i) $y \in \partial u(x)$,

(ii) $x \in \partial u^{*}(y)$,

(iii) $\langle x, y\rangle=u(x)+u^{*}(y)$,

(iv) $x \in \operatorname{argmax}_{z \in \mathbb{R}^{n}}(\langle y, z\rangle-u(z))$,

(v) $y \in \operatorname{argmax}_{z \in \mathbb{R}^{n}}\left(\langle x, z\rangle-u^{*}(z)\right)$.

Here $\operatorname{argmax}_{z \in V} v(z)$ denotes the set of points in the set $V$ at which the function values of $v$ are maximized on $V$. 


\section{LIPSCHITZ REGULARIZATION}

For a function $u \in \operatorname{Conv}\left(\mathbb{R}^{n}\right)$ and $r>0$, we consider its Pasch-Hausdorff envelope or Lipschitz regularization

$$
\operatorname{reg}_{r} u=\left(u^{*}+I_{B_{1 / r}^{n}}\right)^{*}
$$

(see [20,21] and [34, Example 9.11]).

Remark. Given $u \in \operatorname{Conv}\left(\mathbb{R}^{n}\right)$ and $r>0$, the Lipschitz regularization $\operatorname{reg}_{r} u$ admits the following equivalent definition

$$
\operatorname{reg}_{r} u(x)=\sup \left\{w(x): w \in \operatorname{Aff}\left(\mathbb{R}^{n}\right), w \leq u \text { in } \mathbb{R}^{n},|\nabla w| \leq 1 / r\right\} .
$$

In other words, the graph of $\operatorname{reg}_{r} u$ is the envelope of all supporting hyperplanes to the graph of $u$, with slope bounded by $1 / r$. This can be also rephrased as follows: $\operatorname{reg}_{r} u$ is the largest convex function which is smaller than $u$ and has Lipschitz constant bounded by $1 / r$ (for sufficiently large $r$ ). For brevity, we omit the proof of the equivalence of these definitions.

Proposition 4.1. For $u \in \operatorname{Conv}\left(\mathbb{R}^{n}\right)$ and $r>0$, the Lipschitz regularization has the following properties.

(i) For $r>0$, the function $\operatorname{reg}_{r} u$ is convex in $\mathbb{R}^{n}$. There exists $r_{0}>0$ such that $\operatorname{reg}_{r} u(x)>-\infty$ for every $0<r \leq r_{0}$ and $x \in \mathbb{R}^{n}$.

(ii) For $r>0$, we have $\operatorname{reg}_{r} u<+\infty$ on $\mathbb{R}^{n}$.

(iii) For $0<r<t$, we have $\operatorname{reg}_{t} u(x) \leq \operatorname{reg}_{r} u(x) \leq u(x)$ for every $x \in \mathbb{R}^{n}$.

(iv) As $r \rightarrow 0$, the functions reg $_{r} u$ epi-converge to $u$.

(v) For every $x \in \mathbb{R}^{n}$, we have $\lim _{r \rightarrow 0} \operatorname{reg}_{r} u(x)=u(x)$.

(vi) If $x \in \operatorname{dom}(u)$ and $y \in \partial u(x)$, then $\operatorname{reg}_{r} u(x)=u(x)$ and $\partial \operatorname{reg}_{r} u(x)=\partial u(x) \cap B_{1 / r}^{n}$ for $|y|<\frac{1}{r}$.

Proof. (i) Since $\operatorname{reg}_{r} u$ is defined as the conjugate of a convex function, it is convex itself. Furthermore, since $u^{*} \not \equiv+\infty$, there exists $r_{0}>0$ such that $\operatorname{dom}\left(u^{*}\right) \cap B_{1 / r}^{n} \neq \emptyset$ for every $0<r \leq r_{0}$ and therefore $u^{*}+I_{B_{1 / s}^{n}} \in \operatorname{Conv}\left(\mathbb{R}^{n}\right)$. Hence, Proposition 3.3 implies that $\operatorname{reg}_{r} u \in \operatorname{Conv}\left(\mathbb{R}^{n}\right)$ for every $0<\leq r_{0}$.

(ii) It follows from the definition of the convex conjugate, that

$$
\operatorname{reg}_{r} u(x)=\sup _{y \in \mathbb{R}^{n}}\left(\langle x, y\rangle-\left(u^{*}(y)+I_{B_{1 / r}^{n}}(y)\right)\right)=\sup _{y \in B_{1 / r}^{n}}\left(\langle x, y\rangle-u^{*}(y)\right)<+\infty .
$$

(iii) This follows from the fact that convex conjugation is order reversing, that is, if $u \leq v$ pointwise, then $u^{*} \geq v^{*}$ pointwise.

(iv) By Proposition 3.5, it is enough to show that $u^{*}+I_{B_{1 / r}^{n}}$ is epi-convergent to $u^{*}$, which follows from the properties of epi-convergence (see also [34, Theorem 7.46]).

(v) By (iii), the function $\operatorname{reg}_{r} u(x)$ is bounded above by $u(x)$ and decreasing in $r$. Hence, $\lim _{r \rightarrow 0} \operatorname{reg}_{r} u(x)$ exists in $(-\infty,+\infty]$. Together with (iv) and the definition of epi-convergence, this gives

$$
u(x) \leq \lim _{s \rightarrow 0} \operatorname{reg}_{r} u(x) \leq u(x) .
$$

(vi) For $x \in \operatorname{dom}(u)$, we have by Lemma 3.7

$$
\begin{aligned}
y \in \partial u(x), \quad|y| \leq \frac{1}{s} & \Longleftrightarrow y \in \operatorname{argmax}_{z \in B_{1 / r}^{n}}\left(\langle x, z\rangle-u^{*}(z)\right) \\
& \Longleftrightarrow y \in \operatorname{argmax}_{z \in \mathbb{R}^{n}}\left(\langle x, z\rangle-\left(u^{*}(z)+I_{B_{1 / r}^{n}}(z)\right)\right) \\
& \Longleftrightarrow y \in \operatorname{argmax}_{z \in \mathbb{R}^{n}}\left(\langle x, z\rangle-\left(\operatorname{reg}_{r} u\right)^{*}(z)\right) \\
& \Longleftrightarrow y \in \partial \operatorname{reg}_{r} u(x) .
\end{aligned}
$$


Hence, for $y \in \partial u(x)$ with $|y|<\frac{1}{r}$,

$$
u(x)=\sup _{z \in B_{1 / r}^{n}}\left(\langle x, z\rangle-u^{*}(z)\right)=\sup _{z \in \mathbb{R}^{n}}\left(\langle x, z\rangle-u^{*}(z)-I_{B_{1 / r}^{n}}(z)\right)=\operatorname{reg}_{r} u(x),
$$

which concludes the proof.

Proposition 4.2. Let $u_{j}, u \in \operatorname{Conv}\left(\mathbb{R}^{n}\right)$. If $u_{j}$ epi-converges to $u$, then $\operatorname{reg}_{r} u_{j}$ epi-converges to $\operatorname{reg}_{r} u$ as $j \rightarrow+\infty$ for sufficiently small $r>0$.

Proof. By Proposition 3.5, it is enough to prove that given a sequence $v_{j} \in \operatorname{Conv}\left(\mathbb{R}^{n}\right)$ that is epiconvergent to $v \in \operatorname{Conv}\left(\mathbb{R}^{n}\right)$, then, for $s>0$ sufficiently large, the sequence $v_{j}+I_{B_{s}^{n}}$ epi-converges to $v+I_{B_{s}^{n}}$.

Let us fix $s>0$ such that there exists $\bar{x} \in \operatorname{dom}(v) \cap B_{s / 4}^{n}$. By epi-convergence, we may assume that for every $j$ there exists $\bar{x}_{j} \in B_{s / 2}^{n}$ such that $v_{j}\left(\bar{x}_{j}\right) \leq 2 v(\bar{x})$. Let $x \in \mathbb{R}^{n}$ and $x_{j}$ be a sequence converging to $x$. The inequality

$$
\liminf _{j \rightarrow+\infty}\left(v_{j}+I_{B_{s}^{n}}\right)\left(x_{j}\right) \geq\left(v+I_{B_{s}^{n}}\right)(x)
$$

follows easily from the epi-convergence of the sequence $v_{j}$ to $v$. To conclude, we have to show that there exists a specific sequence $x_{j}$ converging to $x$ and such that

$$
\lim _{j \rightarrow+\infty}\left(v_{j}+I_{B_{s}^{n}}\right)\left(x_{j}\right)=\left(v+I_{B_{s}^{n}}\right)(x) .
$$

If either $|x|<s$ or $|x|>s$, this is again a straightforward consequence of epi-convergence. So assume that $|x|=s$ and let $x_{j}$ be a sequence such that

$$
\lim _{j \rightarrow+\infty} v_{j}\left(x_{j}\right)=v(x)
$$

If all but a finite number of the elements of $x_{j}$ belong to $B_{s}^{n}$, then (4.1) follows. Hence we may assume that $\left|x_{j}\right|>s$ for every $j$. Let $\hat{x}_{j}$ be the intersection of the segment joining $x_{j}$ and $\bar{x}_{j}$ with $\operatorname{bd}\left(B_{s}^{n}\right)$. Then

$$
\hat{x}_{j}=\left(1-t_{j}\right) \bar{x}_{j}+t_{j} x_{j}
$$

for a suitable $t_{j} \in[0,1]$. As $|x|=\left|x_{j}\right|=s$ and $\left|\bar{x}_{j}\right| \leq s / 2$ for every $j$, up to extracting a subsequence, we may assume that $t_{j} \rightarrow 1$ as $j \rightarrow+\infty$, so that, in particular

$$
\lim _{j \rightarrow+\infty} \hat{x}_{j}=x .
$$

Hence

$$
\liminf _{j \rightarrow+\infty}\left(v_{j}+I_{B_{s}^{n}}\right)\left(\hat{x}_{j}\right)=\liminf _{j \rightarrow+\infty} v_{j}\left(\hat{x}_{j}\right) \geq v(x)=\left(v+I_{B_{s}^{n}}\right)(x) .
$$

On the other hand, by convexity

$$
\left(v_{j}+I_{B_{r}^{n}}\right)\left(\hat{x}_{j}\right)=v_{j}\left(\hat{x}_{j}\right) \leq\left(1-t_{j}\right) v_{j}\left(\bar{x}_{j}\right)+t_{j} v_{j}\left(x_{j}\right)
$$

for $j \in \mathbb{N}$. Passing to the limit as $j \rightarrow+\infty$ (and recalling that the sequence $v_{j}\left(\bar{x}_{j}\right)$ is bounded from above), we obtain

$$
\limsup _{j \rightarrow+\infty}\left(v_{j}+I_{B_{s}^{n}}\right)\left(\hat{x}_{j}\right) \leq v(x)=\left(v+I_{B_{s}^{n}}\right)(x)
$$

which concludes the proof.

Proposition 4.3. Let $u, v \in \operatorname{Conv}\left(\mathbb{R}^{n}\right)$. If $u \wedge v \in \operatorname{Conv}\left(\mathbb{R}^{n}\right)$, then

$$
\operatorname{reg}_{r}(u \wedge v)=\operatorname{reg}_{r} u \wedge \operatorname{reg}_{r} v, \quad \operatorname{reg}_{r}(u \vee v)=\operatorname{reg}_{r} u \vee \operatorname{reg}_{r} v
$$

for sufficiently small $r>0$. 
Proof. By Proposition 3.4, the function $u^{*} \wedge v^{*}$ is convex. Hence,

$$
\left(\operatorname{reg}_{r} u\right)^{*} \wedge\left(\operatorname{reg}_{r} v\right)^{*}=\left(u^{*}+I_{B_{1 / r}^{n}}\right) \wedge\left(v^{*}+I_{B_{1 / r}^{n}}\right)=\left(u^{*} \wedge v^{*}\right)+I_{B_{1 / r}^{n}}
$$

is convex as well, and this implies that $\operatorname{reg}_{r} u \wedge \operatorname{reg}_{r} v$ is convex. Consequently

$$
\begin{aligned}
\operatorname{reg}_{r}(u \wedge v) & =\left((u \wedge v)^{*}+I_{B_{1 / r}^{n}}\right)^{*} \\
& =\left(u^{*} \vee v^{*}+I_{B_{1 / r}^{n}}\right)^{*} \\
& =\left(\left(u^{*}+I_{B_{1 / r}^{n}}\right) \vee\left(v^{*}+I_{B_{1 / r}^{n}}\right)\right)^{*} \\
& =\left(\left(\operatorname{reg}_{r} u\right)^{*} \vee\left(\operatorname{reg}_{r} v\right)^{*}\right)^{*} \\
& =\operatorname{reg}_{r} u \wedge \operatorname{reg}_{r} u .
\end{aligned}
$$

The second equation is proved analogously.

\section{INCLUSION-EXCLUSION PROPERTIES OF THE SUbGRAdiEnt MAP}

In this section we will prove the following result.

Theorem 5.1. Let $u, v \in \operatorname{Conv}\left(\mathbb{R}^{n}\right)$. If $u \wedge v \in \operatorname{Conv}\left(\mathbb{R}^{n}\right)$, then

$$
\begin{aligned}
& \partial(u \vee v)(x) \cup \partial(u \wedge v)(x)=\partial u(x) \cup \partial v(x), \\
& \partial(u \vee v)(x) \cap \partial(u \wedge v)(x)=\partial u(x) \cap \partial v(x)
\end{aligned}
$$

for every $x \in \mathbb{R}^{n}$.

The proof will require some preliminary steps.

Lemma 5.2. Let $C_{1}$ and $C_{2}$ be non-empty, closed and convex subsets of $\mathbb{R}^{n}$. If $C_{1} \cup C_{2}$ is convex, then $C_{1} \cap C_{2} \neq \emptyset$.

Proof. Assume that $C_{1} \cap C_{2}=\emptyset$. Let $B$ be a ball centered at the origin such that $K_{i}:=B \cap C_{i} \neq \emptyset$ for $i=1,2$. The convex bodies $K_{1}$ and $K_{2}$ are disjoint, and hence they can be strongly separated (see [35, Theorem 1.3.7]) which implies in particular that their union is not convex. On the other hand $K_{1} \cup K_{2}=\left(C_{1} \cup C_{2}\right) \cap B$ must be convex.

Note that the claim of the previous lemma fails to be true without assuming the sets to be closed.

Lemma 5.3. Let $u, v \in \operatorname{Conv}\left(\mathbb{R}^{n}\right)$. If $u \wedge v \in \operatorname{Conv}\left(\mathbb{R}^{n}\right)$ and $u \vee v \geq 0$ in $\mathbb{R}^{n}$, then $u \geq 0$ or $v \geq 0$ in $\mathbb{R}^{n}$.

Proof. By contradiction, assume that there exist $x_{1}, x_{2} \in \mathbb{R}^{n}$ such that $c:=u\left(x_{1}\right) \vee v\left(x_{2}\right)<0$. Let

$$
C_{1}=\left\{x \in \mathbb{R}^{n}: u(x) \leq c\right\}, \quad C_{2}=\left\{x \in \mathbb{R}^{n}: v(x) \leq c\right\}
$$

These are non-empty, closed convex sets. Moreover

$$
\left\{x \in \mathbb{R}^{n}:(u \wedge v)(x) \leq c\right\}=C_{1} \cup C_{2},
$$

that is, their union is convex. By the previous lemma, there exists $x \in C_{1} \cap C_{2}$; on the other hand this leads to

$$
(u \vee v)(x) \leq c<0
$$

which is a contradiction.

We proceed with a one-dimensional result.

Lemma 5.4. Let $u, v: \mathbb{R} \rightarrow \mathbb{R} \cup\{+\infty\}$ be convex and l.s.c. and assume that $u \wedge v$ is convex. If $\bar{x} \in \mathbb{R}$ is such that $u(\bar{x})<v(\bar{x})$, then there exists $\delta>0$ such that $u(x) \leq v(x)$ for all $x \in[\bar{x}-\delta, \bar{x}+\delta]$. 
Proof. Clearly $\bar{x} \in \operatorname{dom}(u)$. If $\bar{x}$ is in the interior of $\operatorname{dom}(u)$, then $u$ is continuous at $\bar{x}$ and the statement follows. If $\bar{x}$ is a boundary point of $\operatorname{dom}(u)$ and $u(x)=+\infty$ for $x>\bar{x}$, say, then the convexity of $u \wedge v$ implies that $v(x)=+\infty$ for $x>\bar{x}$, too.

Lemma 5.5. Let $u, v \in \operatorname{Conv}\left(\mathbb{R}^{n}\right)$ and $u \wedge v \in \operatorname{Conv}\left(\mathbb{R}^{n}\right)$. If $x \in \mathbb{R}^{n}$ is such that $u(x)<v(x)$, then $(u \wedge v)^{\prime}(x ; y)=u^{\prime}(x ; y)$ for every $y \in \mathbb{R}^{n}$. Moreover, if $v(x)$ is finite, then $(u \vee v)^{\prime}(x ; y)=v^{\prime}(x ; y)$ for every $y \in \mathbb{R}^{n}$.

Proof. The statement is trivially true for $y=0$; so we assume $y \neq 0$. Consider the line $L$ passing through $x$ and parallel to $y$. The restrictions of $u$ and $v$ to $L$ are l.s.c. convex functions of one variable, such that their minimum is convex. By the previous lemma, for $|t|$ sufficiently small,

$$
(u \wedge v)(x+t y)=u(x+t y), \quad(u \vee v)(x+t y)=v(x+t y) .
$$

The conclusion follows immediately.

Proof of Theorem 5.1. First, if $u(x)<v(x)$, then Lemma 3.6 and Lemma 5.5 imply that

$$
\partial(u \wedge v)(x)=\partial u(x) \text { and } \partial(u \vee v)(x)=\partial v(x) .
$$

Second, if $u(x)=v(x)$, we show that

$$
\begin{aligned}
& \partial(u \vee v)(x)=\partial u(x) \cup \partial v(x), \\
& \partial(u \wedge v)(x)=\partial u(x) \cap \partial v(x) .
\end{aligned}
$$

Indeed, if $u(x)=v(x)=+\infty$, then all subdifferentials are empty. So assume that $x \in \operatorname{dom}(u) \cap \operatorname{dom}(v)$. The first equation in (5.2) is a straightforward consequence of the definition of subdifferentials and the equality $u(x)=v(x)$. Concerning the second equation, let

$$
C=\partial(u \vee v)(x), \quad D=\partial u(x) \cup \partial v(x) .
$$

If $y \in D$, then we may assume that $y \in \partial u(x)$, which yields, for every $z \in \mathbb{R}^{n}$,

$$
(u \vee v)(z) \geq u(z) \geq u(x)+\langle z-x, y\rangle=(u \vee v)(x)+\langle z-x, y\rangle,
$$

that is, $y \in C$. Now assume that $y \in C$. Define $w \in \operatorname{Conv}\left(\mathbb{R}^{n}\right)$ as

$$
w(z)=u(x)+\langle z-x, y\rangle,
$$

and set $\bar{u}=u-w$ and $\bar{v}=v-w$. Then $\bar{u}, \bar{v} \in \operatorname{Conv}\left(\mathbb{R}^{n}\right)$ and

$$
\bar{u} \wedge \bar{v}=(u \wedge v)-w, \quad \bar{u} \vee \bar{v}=(u \vee v)-w .
$$

In particular, $\bar{u} \wedge \bar{v} \in \operatorname{Conv}\left(\mathbb{R}^{n}\right)$. Now, $y \in \partial(u \vee v)(x)$ implies $0 \in \partial(\bar{u} \vee \bar{v})(x)$; moreover $(\bar{u} \vee \bar{v})(x)=0$. Hence $\bar{u} \vee \bar{v} \geq 0$ in $\mathbb{R}^{n}$. By Lemma 5.3, one of the two functions $\bar{u}$ and $\bar{v}$ is non-negative in $\mathbb{R}^{n}$. Assuming for instance $\bar{u} \geq 0$, we obtain, as $\bar{u}$ vanishes at $x$, that $0 \in \partial \bar{u}(x)$ which implies $y \in \partial u(x)$, that is, $y \in D$.

Finally, note that (5.1) and (5.2) imply the statement of the theorem.

\section{The Graph of the Subgradient Map and Parallel Sets of Functions}

We start by recalling two important definitions.

Definition 6.1. For $u \in \operatorname{Conv}\left(\mathbb{R}^{n}\right)$, the graph of the subdifferential map of $u$ is defined as

$$
\Gamma_{u}:=\left\{(x, y): x \in \mathbb{R}^{n}, y \in \partial u(x)\right\} .
$$


Next we define parallel sets of a convex function.

Definition 6.2. Let $u \in \operatorname{Conv}\left(\mathbb{R}^{n}\right)$ and $\eta \subset \mathbb{R}^{n} \times \mathbb{R}^{n}$. For $s \geq 0$, we set

$$
P_{s}(u, \eta)=\left\{x+s y:(x, y) \in \eta \cap \Gamma_{u}\right\} .
$$

Note that for $s=0$ we have $P_{0}(u, \eta)=\pi_{1}\left(\eta \cap \Gamma_{u}\right)$.

6.1. Inclusion-exclusion results. The following result is an immediate consequence of Theorem 5.1 ,

Proposition 6.3. Let $u, v \in \operatorname{Conv}\left(\mathbb{R}^{n}\right)$. If $u \wedge v \in \operatorname{Conv}\left(\mathbb{R}^{n}\right)$, then

$$
\Gamma_{u \vee v} \cap \Gamma_{u \wedge v}=\Gamma_{u} \cap \Gamma_{v} \text { and } \Gamma_{u \vee v} \cup \Gamma_{u \wedge v}=\Gamma_{u} \cup \Gamma_{v} .
$$

Next, we establish a corresponding result for the parallel sets of the subdifferential graph.

Proposition 6.4. Let $u, v \in \operatorname{Conv}\left(\mathbb{R}^{n}\right)$. If $u \wedge v \in \operatorname{Conv}\left(\mathbb{R}^{n}\right)$, then

$$
\begin{gathered}
P_{s}(u \vee v, \eta) \cup P_{s}(u \wedge v, \eta)=P_{s}(u, \eta) \cup P_{s}(v, \eta), \\
P_{s}(u \vee v, \eta) \cap P_{s}(u \wedge v, \eta)=P_{s}(u, \eta) \cap P_{s}(v, \eta)
\end{gathered}
$$

for every $\eta \subset \mathbb{R}^{n} \times \mathbb{R}^{n}$ and $s \geq 0$.

Proof. The case $s=0$ follows easily from the previous proposition. For $s>0$, we have

$$
\begin{aligned}
P_{s}(u \vee v, \eta) \cup P_{s}(u \wedge v, \eta) & =\left\{x+s y:(x, y) \in \eta \cap \Gamma_{u \vee v}\right\} \cup\left\{x+s y:(x, y) \in \eta \cap \Gamma_{u \wedge v}\right\} \\
& =\left\{x+s y:(x, y) \in \eta \cap\left(\Gamma_{u \vee v} \cup \Gamma_{u \wedge v}\right)\right\} \\
& =\left\{x+s y:(x, y) \in \eta \cap\left(\Gamma_{u} \cup \Gamma_{v}\right)\right\} \\
& =\left\{x+s y:(x, y) \in \eta \cap \Gamma_{u}\right\} \cup\left\{x+s y:(x, y) \in \eta \cap \Gamma_{v}\right\} \\
& =P_{s}(u, \eta) \cup P_{s}(v, \eta) .
\end{aligned}
$$

This proves 6.1). The proof of (6.2) is analogous.

6.2. An auxiliary proposition. Given $u \in \operatorname{Conv}\left(\mathbb{R}^{n}\right)$ and $r>0$, we set

$$
\Gamma_{u}^{r}:=\Gamma_{u} \cap\left\{(x, y) \in \mathbb{R}^{2 n}:|y| \leq r\right\} .
$$

We require the following properties of the Lipschitz regularization of a function $u \in \operatorname{Conv}\left(\mathbb{R}^{n}\right)$ (in addition to those presented in Section 4).

Proposition 6.5. If $u \in \operatorname{Conv}\left(\mathbb{R}^{n}\right)$ and $r>0$, then $u_{r}=\operatorname{reg}_{r} u$ has the following properties.

(i) $\Gamma_{u}^{r} \subset \Gamma_{u_{r}}$.

(ii) For every $\eta \subset \Gamma_{u}^{r}$ and $s \geq 0$, we have $P_{s}(u, \eta)=P_{s}\left(u_{r}, \eta\right)$.

Proof. By (vi) from Proposition 4.1, it follows from $(x, y) \in \Gamma_{u}^{r}$ that $u_{r}(x)=u(x)$ and $\partial u_{r}(x)=$ $\partial u(x) \cap\left\{y \in \mathbb{R}^{n}:|y| \leq r\right\}$, which implies (ii) and therefore (ii).

\section{Hessian Measures}

We recall and extend the definition of Hessian measures. First, we establish the following result.

Theorem 7.1. For $u \in \operatorname{Conv}\left(\mathbb{R}^{n}\right)$, there are non-negative Borel measures $\Theta_{0}(u, \cdot), \ldots, \Theta_{n}(u, \cdot)$ on $\mathbb{R}^{2 n}$ such that

$$
\mathcal{H}^{n}\left(P_{s}(u, \eta)\right)=\sum_{i=0}^{n}\left(\begin{array}{l}
n \\
i
\end{array}\right) s^{i} \Theta_{n-i}(u, \eta)
$$

for every $\eta \in \mathcal{B}\left(\mathbb{R}^{2 n}\right)$ and $s \geq 0$, 
We will call the measures $\Theta_{i}(u, \cdot)$ for $i=0, \ldots, n$ the Hessian measures of $u$. The proof makes use of the following result from [10] (see Theorem 3.1; also see [11, Section 5]). For an open subset $U$ of $\mathbb{R}^{n}$, we denote by $\mathcal{B}(U)$ the family of Borel subsets of $U$.

Theorem 7.2. If $u \in \operatorname{Conv}\left(\mathbb{R}^{n}\right)$ and $U:=\operatorname{int}(\operatorname{dom}(u))$ is not empty, then there are non-negative Borel measures $\Theta_{0}(u, \cdot), \ldots, \Theta_{n}(u, \cdot)$ on $U \times \mathbb{R}^{n}$ such that (7.1) holds for every $\eta \in \mathcal{B}\left(U \times \mathbb{R}^{n}\right)$ and $s \geq 0$.

7.1. Proof of Theorem 7.1. Let $r>0$ and set $u_{r}=\operatorname{reg}_{r} u$. For $\eta \in \mathcal{B}\left(\mathbb{R}^{2 n}\right)$ with $\eta \subset \mathbb{R}^{n} \times B_{r}^{n}$, it follows from (ii) of Proposition 6.5 that $P_{s}(u, \eta)=P_{s}\left(u_{r}, \eta \cap \Gamma_{u}\right)$. Hence, by Theorem 7.2,

$$
\mathcal{H}^{n}\left(P_{s}(u, \eta)\right)=\sum_{i=0}^{n}\left(\begin{array}{l}
n \\
i
\end{array}\right) s^{i} \Theta_{n-i}\left(u_{r}, \eta \cap \Gamma_{u}\right) .
$$

In this way we have proved the following fact: for every $r>0$, there exists a set of $(n+1)$ Borel measures on $\mathcal{B}\left(\mathbb{R}^{n} \times B_{r}^{n}\right)$, namely $\Theta_{i, r}\left(u, \cap \cap \Gamma_{u}\right):=\Theta_{i}\left(u_{r}, \cdot \cap \Gamma_{u}\right)$, such that (7.1) holds for every $\eta \in \mathcal{B}\left(\mathbb{R}^{n} \times B_{r}^{n}\right)$ and $s \geq 0$. If $r^{\prime} \geq r \geq 0$, as $\mathcal{B}\left(\mathbb{R}^{n} \times B_{r^{\prime}}^{n}\right) \supset \mathcal{B}\left(\mathbb{R}^{n} \times B_{r}^{n}\right)$, we easily get

$$
\Theta_{i, r}(u, \eta)=\Theta_{i, r^{\prime}}(u, \eta)
$$

for all $\eta \in \mathcal{B}\left(\mathbb{R}^{n} \times B_{r}^{n}\right)$ and $i=0, \ldots, n$. Such measures can be extended to $\mathcal{B}\left(\mathbb{R}^{2 n}\right)$ by a standard procedure, as they are non-negative:

$$
\Theta_{i}(u, \eta)=\lim _{r \rightarrow+\infty} \Theta_{i, r}\left(u, \eta \cap\left(\mathbb{R}^{n} \times B_{r}^{n}\right)\right)
$$

for $\eta \in \mathcal{B}\left(\mathbb{R}^{2 n}\right)$ and $i=0, \ldots, n$.

The validity of the Steiner formula (7.1) is preserved as

$$
P_{s}(u, \eta)=\bigcup_{r \geq 0} P_{s}\left(u, \eta \cap \Gamma_{u}^{r}\right)
$$

so that

$$
\mathcal{H}^{n}\left(P_{s}(u, \eta)\right)=\lim _{r \rightarrow+\infty} \mathcal{H}^{n}\left(P_{s}\left(u, \eta \cap\left(\mathbb{R}^{n} \times B_{r}^{n}\right)\right)\right)
$$

for every $\eta \in \mathcal{B}\left(\mathbb{R}^{2 n}\right)$ and $s \geq 0$.

Remark. The notion of Hessian measure is clearly of local nature. Let $u$ and $v$ be real-valued convex functions defined in an open convex set $U \subset \mathbb{R}^{n}$. Assume that $\partial u(x)=\partial v(x)$ for every $x \in \beta \in \mathcal{B}(U)$. If $\eta \in \mathcal{B}\left(U \times \mathbb{R}^{n}\right)$ is such that $\pi_{1}(\eta) \subset \beta$, then

$$
P_{s}(u, \eta)=P_{s}(v, \eta)
$$

for every $s \geq 0$. Hence

$$
\Theta_{i}(u, \eta)=\Theta_{i}(v, \eta)
$$

for $i=0, \ldots, n$.

Remark. For $u \in \operatorname{Conv}\left(\mathbb{R}^{n}\right)$, the support of the Hessian measures of $u$ is contained in $\Gamma_{u}$. Indeed, if $\eta \subset \mathbb{R}^{n} \backslash \Gamma_{u}$, then for every $(x, y) \in \eta$, we have $\partial u(x)=\emptyset$. Hence, for $s \geq 0$, we have $P_{s}(u, \eta)=\emptyset$, which implies that

$$
0=\mathcal{H}^{n}\left(P_{s}(u, \eta)\right)=\sum_{i=0}^{n}\left(\begin{array}{c}
n \\
i
\end{array}\right) s^{i} \Theta_{n-i}(u, \eta) .
$$

As Hessian measures are non-negative, we get $\Theta_{i}(u, \eta)=0$ for every $i=0, \ldots, n$. 
Remark. Hessian measures are locally finite. Indeed, assume that $|(x, y)| \leq r$ for every $(x, y) \in \eta$, for some $r>0$. As each $\Theta_{i}(u, \cdot)$ is non-negative, by the Steiner formula (for $s=1$ ) we get

$$
\left(\begin{array}{l}
n \\
i
\end{array}\right) \Theta_{i}(u, \eta) \leq \mathcal{H}^{n}\left(P_{1}(u, \eta)\right) .
$$

On the other hand, we have $P_{1}(u, \eta) \subset\left\{z \in \mathbb{R}^{n}:|z| \leq 2 r\right\}$. Hence, for $i=0, \ldots, n$,

$$
\Theta_{i}(u, \eta) \leq c(n) \operatorname{diam}(\eta)^{n}
$$

for every $u \in \operatorname{Conv}\left(\mathbb{R}^{n}\right)$ and $\eta \in \mathcal{B}\left(\mathbb{R}^{2 n}\right)$, where $c(n)$ only depends on the dimension and diam stands for diameter.

7.2. Continuity. We show that Hessian measures are weakly continuous with respect to epi-convergence.

Theorem 7.3. Let $u_{k}$ be a sequence in $\operatorname{Conv}\left(\mathbb{R}^{n}\right)$. If $u_{k}$ epi-converges to $u \in \operatorname{Conv}\left(\mathbb{R}^{n}\right)$, then the sequence of measures $\Theta_{i}\left(u_{k}, \cdot\right)$ converges weakly to $\Theta_{i}(u, \cdot)$ as $k \rightarrow+\infty$ for every $i=0, \ldots, n$.

Proof. First, using the Steiner formula (7.1), it is easy to see that it is sufficient to prove for every $s>0$ that the sequence of measures $\eta \mapsto \mathcal{H}^{n}\left(P_{s}\left(u_{k}, \eta\right)\right)$ converges weakly to the measure $\eta \mapsto \mathcal{H}^{n}\left(P_{s}(u, \eta)\right)$ as $k \rightarrow+\infty$.

Second, to establish the previous claim we prove that if $\eta \subset \mathbb{R}^{2 n}$ is compact, then

$$
\limsup _{k \rightarrow+\infty} \mathcal{H}^{n}\left(P_{s}\left(u_{k}, \eta\right)\right) \leq \mathcal{H}^{n}\left(P_{s}(u, \eta)\right)
$$

and, if $\eta \subset \mathbb{R}^{2 n}$ is open, then

$$
\liminf _{k \rightarrow+\infty} \mathcal{H}^{n}\left(P_{s}\left(u_{k}, \eta\right)\right) \geq \mathcal{H}^{n}\left(P_{s}(u, \eta)\right)
$$

Third, we prove (7.3). To simplify notation, for fixed $\eta \subset \mathbb{R}^{2 n}$ and $s>0$, we set $A=P_{s}(u, \eta)$ and $A_{k}=P_{s}\left(u_{k}, \eta\right)$ for $k \in \mathbb{N}$. As $\eta$ is compact, $A$ is compact as well. Indeed, it is obviously bounded. To prove that it is closed, let $z_{j}$ be a sequence in $A$, converging to some $\bar{z} \in \mathbb{R}^{n}$. Then there exists a sequence $x_{j}$ in $\mathbb{R}^{n}$ and a sequence $y_{j}$ such that $y_{j} \in \partial u\left(x_{j}\right)$ and $\left(x_{j}, y_{j}\right) \in \eta$ for every $j$ and $z_{j}=x_{j}+s y_{j}$. By compactness, we may assume that $x_{j}$ and $y_{j}$ converge to some $\bar{x} \in \mathbb{R}^{n}$ and $\bar{y} \in \mathbb{R}^{n}$, respectively, with $(\bar{x}, \bar{y}) \in \eta$. For every $x \in \mathbb{R}^{n}$, we have

$$
u(x) \geq u\left(x_{j}\right)+\left\langle x-x_{j}, y_{j}\right\rangle .
$$

Passing to the limit and using the lower semicontinuity of $u$, we obtain

$$
u(x) \geq u(\bar{x})+\langle x-\bar{x}, \bar{y}\rangle
$$

for all $x \in \mathbb{R}^{n}$. Hence $\bar{y} \in \partial u(\bar{x})$, so that $\bar{z} \in A$.

Next, we prove that for every $\varepsilon>0$ there exists $\bar{k} \in \mathbb{N}$ such that

$$
A_{k} \subset(A)_{\varepsilon}
$$

for all $k \geq \bar{k}$, where $A_{\varepsilon}:=\left\{x \in \mathbb{R}^{n}: \operatorname{dist}(x, A) \leq \varepsilon\right\}$ and $\operatorname{dist}(x, A)=\inf \{|x-y|: y \in A\}$. Note that (7.3) follows from (7.5), as $A=\bigcap_{\varepsilon>0} A_{\varepsilon}$ implies that $\mathcal{H}^{n}(A)=\lim _{\varepsilon \rightarrow 0^{+}} \mathcal{H}^{n}\left(A_{\varepsilon}\right)$, where the first equality holds because $A$ is closed. We argue by contradiction. Assume that there exists $\varepsilon>0$, a sequence $x_{k} \in \mathbb{R}^{n}$ and a sequence $y_{k} \in \mathbb{R}^{n}$, with $y_{k} \in \partial u_{k}\left(x_{k}\right)$ and $\left(x_{k}, y_{k}\right) \in \eta$ for every $k \in \mathbb{N}$, such that

$$
\operatorname{dist}\left(x_{k}+s y_{k}, A\right) \geq \varepsilon
$$


for all $k \in \mathbb{N}$. By compactness, we may assume that the sequences $x_{k}$ and $y_{k}$ converge to $\bar{x}$ and $\bar{y} \in \mathbb{R}^{n}$, respectively, with $(\bar{x}, \bar{y}) \in \eta$. Let $x \in \mathbb{R}^{n}$; by the definition of epi-convergence, there exists a sequence $\hat{x}_{k}$ such that

$$
\lim _{k \rightarrow+\infty} u_{k}\left(\hat{x}_{k}\right)=u(x)
$$

Moreover

$$
\liminf _{k \rightarrow+\infty} u_{k}\left(x_{k}\right) \geq u(\bar{x})
$$

again by epi-convergence. From the inequality

$$
u_{k}\left(\hat{x}_{k}\right) \geq u_{k}\left(x_{k}\right)+\left\langle\hat{x}_{k}-x_{k}, y_{k}\right\rangle
$$

for all $k \in \mathbb{N}$, passing to the limit in $k$, we deduce

$$
u(x) \geq u(\bar{x})+\langle x-\bar{x}, \bar{y}\rangle
$$

for $x \in \mathbb{R}^{n}$, that is, $\bar{y} \in \partial u(\bar{x})$ so that $x_{k}+s y_{k}$ converges to a point of $A$, which contradicts (7.6).

Finally, we prove (7.4). Define $w, w_{k} \in \operatorname{Conv}\left(\mathbb{R}^{n}\right)$ as

$$
w(x)=s u(x)+\frac{|x|^{2}}{2}, \quad w_{k}(x)=s u_{k}(x)+\frac{|x|^{2}}{2},
$$

and note that $\partial w(x)=x+s \partial u(x)$ while $\partial w_{k}(x)=x+s \partial u_{k}(x)$. Let us fix $\bar{x} \in \mathbb{R}^{n}$ and $\bar{z} \in \partial w(x)$. The function $\hat{w} \in \operatorname{Conv}\left(\mathbb{R}^{n}\right)$ defined as

$$
\hat{w}(x)=w(x)-\langle x-\bar{x}, \bar{z}\rangle
$$

has an absolute minimum at $x=\bar{x}$. Consider now, for $k \in \mathbb{N}$, the function $\hat{w}_{k} \in \operatorname{Conv}\left(\mathbb{R}^{n}\right)$ defined as

$$
\hat{w}_{k}(x)=w_{k}(x)-\langle x-\bar{x}, \bar{z}\rangle .
$$

This is a strictly convex and lower semicontinuous function on $\mathbb{R}^{n}$, which verifies

$$
\lim _{|x| \rightarrow+\infty} \hat{w}_{k}(x)=+\infty .
$$

This follows from the fact that $u_{k}$ is bounded from below by an affine function, since its subdifferential is non-empty at least at one point. Hence $w_{k}$ admits a unique (by strict convexity) absolute minimum point $x_{k}$. This implies $0 \in \partial \hat{w}_{k}\left(x_{k}\right)$ which in turn implies that $\bar{z} \in \partial w_{k}\left(x_{k}\right)$.

As $u_{k}$ epi-converges to $u$, the sequence $\hat{w}_{k}$ epi-converges to $\hat{w}$, so that (see for instance Theorem 7.33 in [34])

$$
\lim _{k \rightarrow+\infty} x_{k}=\bar{x} .
$$

We have proved that given $(\bar{x}, \bar{y}) \in \eta \cap \Gamma_{u}$, there exists a sequence $\left(x_{k}, y_{k}\right)$ with $\left(x_{k}, y_{k}\right) \in \Gamma_{u_{k}}$ for every $k$, such that $\bar{x}+s \bar{y}=x_{k}+s y_{k}$ for every $k \in \mathbb{N}$; moreover, $x_{k}$ tends to $\bar{x}$ as $k \rightarrow+\infty$. These conditions imply that $y_{k}$ tends to $\bar{y}$. Hence, as $\eta$ is open, $\left(x_{k}, y_{k}\right)$ is definitively contained in $\eta$. Thus every $z \in P_{s}(u, \eta)$ is contained in $P_{s}\left(u_{k}, \eta\right)$. Consequently,

$$
P_{s}(u, \eta) \subset \bigcup_{i \in \mathbb{N}}\left(\bigcap_{k \geq i} P_{s}\left(u_{k}, \eta\right)\right) .
$$

This easily implies (7.4).

Remark. In the special case of finite functions, the previous theorem was proved in [11, Theorem 1.1]. 
7.3. Integral representations of Hessian measures. Our starting point is the following result, which follows in a rather direct way from results proved in [10]. We recall that a subset $A$ of $\mathbb{R}^{2 n}$ is countably $n$-rectifiable if there exist a countable family $\left\{U_{i}: i \in \mathbb{N}\right\}$ of open subsets of $\mathbb{R}^{n}$ and a countable family $\left\{f_{i}: i \in \mathbb{N}\right\}$ of Lipschitz maps, such that $f_{i}: U_{i} \rightarrow \mathbb{R}^{2 n}$ and

$$
\mathcal{H}^{n}\left(A \backslash \bigcup_{i \in \mathbb{N}} f_{i}\left(U_{i}\right)\right)=0
$$

(see e.g. [17]).

Proposition 7.4. If $u \in \operatorname{Conv}\left(\mathbb{R}^{n}\right)$ is such that $\operatorname{dom}(u)=\mathbb{R}^{n}$, then $\Gamma_{u}$ is a countably $n$-rectifiable set. Moreover, for $i \in\{0, \ldots, n\}$, there exists a non-negative, $\mathcal{H}^{n}$-measurable function $\theta_{i}(u, \cdot): \Gamma_{u} \rightarrow \mathbb{R}$ such that

$$
\Theta_{i}(u, \eta)=\int_{\eta \cap \Gamma_{u}} \theta_{i}(u,(x, y)) \mathrm{d} \mathcal{H}^{n}(x, y)
$$

for every $\eta \in \mathcal{B}\left(\mathbb{R}^{2 n}\right)$.

Proof. The countable $n$-rectifiability of $\Gamma_{u}$ was observed in [10, Section 3].

The set epi $(u)$ is a non-empty, closed, convex subset of $\mathbb{R}^{n+1}$, which does not coincide with $\mathbb{R}^{n+1}$ itself. In particular, its normal bundle, Nor $(\operatorname{epi}(u))$, is well defined:

$$
\operatorname{Nor}(\operatorname{epi}(u))=\left\{(p, q) \in \mathbb{R}^{n+1} \times \mathbb{R}^{n+1}: p \in \operatorname{bd}(\operatorname{epi}(u)), q \in N(\operatorname{epi}(u), p)\right\},
$$

where $N(\operatorname{epi}(u), p)$ denotes the outer normal cone to epi $(u)$ at $p$. Finally, we set

$$
\mathcal{F}_{u}=\operatorname{Nor}(\operatorname{epi}(u)) \cap\left(\left\{(x, u(x)): x \in \mathbb{R}^{n}\right\} \times \mathbb{S}^{n}\right) .
$$

In [10, Section 3] the following facts are proved:

(i) $\mathcal{F}_{u}$ is a countably $n$-rectifiable Borel set;

(ii) there exists an homeomorphism $T: \Gamma_{u} \rightarrow \mathcal{F}_{u}$; moreover $T$ and its inverse are locally Lipschitz.

By [10, Theorem 3.1], each Hessian measure of $u$ admits an integral representation on $\mathcal{F}_{u}$ : there exist $\mathcal{H}^{n}$-measurable, non-negative functions $\bar{\theta}_{i}(u, \cdot): \mathcal{F}_{u} \rightarrow \mathbb{R}$, such that

$$
\Theta_{i}(u, \eta)=\int_{\bar{\eta} \cap \mathcal{F}_{u}} \bar{\theta}_{i}\left(u,(\bar{x}, \bar{y}) \mathrm{d} \mathcal{H}^{n}(\bar{x}, \bar{y}),\right.
$$

for every $\eta \in \mathcal{B}\left(\mathbb{R}^{2 n}\right)$ and $i \in\{0, \ldots, n\}$, where $\bar{\eta}=T\left(\eta \cap \Gamma_{u}\right)$. Now (7.8) follows from performing the change of variables $(\bar{x}, \bar{y})=T(x, y)$ in (7.7) and using the co-area formula (see for instance [17]).

Next we extend the previous result to the general case.

Theorem 7.5. If $u \in \operatorname{Conv}\left(\mathbb{R}^{n}\right)$, then $\Gamma_{u}$ is a countably $n$-rectifiable set. Moreover, for $i \in\{0, \ldots, n\}$, there exists a non-negative, $\mathcal{H}^{n}$-measurable function $\theta_{i}(u, \cdot): \Gamma_{u} \rightarrow \mathbb{R}$ such that

$$
\Theta_{i}(u, \eta)=\int_{\eta \cap \Gamma_{u}} \theta_{i}(u,(x, y)) \mathrm{d} \mathcal{H}^{n}(x, y) .
$$

for every $\eta \in \mathcal{B}\left(\mathbb{R}^{2 n}\right)$.

Proof. For $r>0$, set $u_{r}=\operatorname{reg}_{r} u$ and note that $\operatorname{dom}\left(u_{r}\right)=\mathbb{R}^{n}$. By Proposition 6.5, $\Gamma_{u}^{1 / r} \subset \Gamma_{u_{r}}$. By Proposition 7.4, the set $\Gamma_{u_{r}}$ is countably $n$-rectifiable, and hence the same conclusion is valid for $\Gamma_{u}^{1 / r}$. As

$$
\Gamma_{u}=\bigcup_{r>0} \Gamma_{u}^{1 / r}
$$

we deduce that $\Gamma_{u}$ is countably $n$-rectifiable as well. 
Let us fix $i \in\{0, \ldots, n\}$. As in the proof of Theorem 7.1, we have $P_{s}(u, \eta)=P_{s}\left(u_{r}, \eta\right)$ for every Borel set $\eta \subset \Gamma_{u} \cap\left(\mathbb{R}^{n} \times B_{r}^{n}\right)$. So, in particular

$$
\Theta_{i}(u, \eta)=\Theta_{i}\left(u_{r}, \eta\right)
$$

for every $\eta \in \mathcal{B}\left(\mathbb{R}^{2 n}\right)$ contained in $\Gamma_{u} \cap\left(\mathbb{R}^{n} \times B_{r}^{n}\right)$. By Proposition 7.4, there exists a non-negative $\mathcal{H}^{n}$-measurable function $\theta_{i}\left(u_{r}, \cdot\right)$ defined on $\Gamma_{u_{r}}$ such that

$$
\Theta_{i}(u, \eta)=\Theta_{i}\left(u_{r}, \eta\right)=\int_{\eta \cap \Gamma_{u_{r}}} \theta_{i}\left(u_{r},(x, y)\right) \mathrm{d} \mathcal{H}^{n}(x, y)=\int_{\eta \cap \Gamma_{u}} \theta_{i}\left(u_{r},(x, y)\right) \mathrm{d} \mathcal{H}^{n}(x, y) .
$$

Clearly for $r^{\prime} \geq r$, the function $\theta_{i}\left(u_{r^{\prime}}, \cdot\right)$ extends $\theta_{i}\left(u_{r}, \cdot\right)$. Hence there is a function $\theta_{i}(u, \cdot)$, defined on $\Gamma_{u}$, such that (7.8) holds for every $\eta \in \mathcal{B}\left(\mathbb{R}^{2 n}\right)$ contained in $\mathbb{R}^{n} \times B_{r}^{n}$. The conclusion follows from letting $r \rightarrow+\infty$.

7.4. A second auxiliary result. For future use, we state the following result that follows from the argument used in the proofs in the previous part of this section.

Proposition 7.6. Let $u \in \operatorname{Conv}\left(\mathbb{R}^{n}\right)$. For $r>0$, the function $u_{r}=\operatorname{reg}_{r} u$ has the following properties.

(i) For every $\eta \subset \Gamma_{u}^{r}$ and $i \in\{0, \ldots, n\}$,

$$
\Theta_{i}(u, \eta)=\Theta_{i}\left(u_{r}, \eta\right)
$$

(ii) For $\mathcal{H}^{n}$-a.e. $(x, y) \in \Gamma_{u}^{r}$ and $i \in\{0, \ldots, n\}$,

$$
\theta_{i}(u,(x, y))=\theta_{i}\left(u_{r},(x, y)\right) \text {. }
$$

\section{Further Properties of Hessian Measures}

8.1. Invariance and covariance properties. Let $u \in \operatorname{Conv}\left(\mathbb{R}^{n}\right)$ and $x_{0} \in \mathbb{R}^{n}$; we denote by $u_{x_{0}}$ the composition of $u$ and the translation by $x_{0}$; that is,

$$
u_{x_{0}}(x)=u\left(x-x_{0}\right)
$$

for $x \in \mathbb{R}^{n}$. Clearly, $u_{x_{0}} \in \operatorname{Conv}\left(\mathbb{R}^{n}\right)$. Let $\phi$ be an element of $\mathrm{O}(n)$. Given $u \in \operatorname{Conv}\left(\mathbb{R}^{n}\right)$, we define $u_{\phi}: \mathbb{R}^{n} \rightarrow \mathbb{R}^{n}$ by

$$
u_{\phi}(y)=u\left(\phi^{-1} y\right)
$$

for $\underset{\sim}{y} \in \mathbb{R}^{n}$. As before, we have $u_{\phi} \in \operatorname{Conv}\left(\mathbb{R}^{n}\right)$. For $\phi \in \mathrm{O}(n)$, we also define $\tilde{\phi}: \mathbb{R}^{n} \times \mathbb{R}^{n} \rightarrow \mathbb{R}^{n} \times \mathbb{R}^{n}$ by $\tilde{\phi}(x, y)=(\phi x, \phi y)$.

We establish the following covariance properties of Hessian measures.

Proposition 8.1. Let $u \in \operatorname{Conv}\left(\mathbb{R}^{n}\right)$. For $x_{0} \in \mathbb{R}^{n}$ and $\phi \in \mathrm{O}(n)$,

$$
\begin{aligned}
& \Theta_{i}\left(u_{x_{0}}, \eta\right)=\Theta_{i}\left(u, \eta+\left(x_{0}, 0\right)\right), \\
& \Theta_{i}\left(u_{\phi}, \eta\right)=\Theta_{i}\left(u, \tilde{\phi}^{-1} \eta\right) .
\end{aligned}
$$

for every $\eta \in \mathcal{B}\left(\mathbb{R}^{n} \times \mathbb{R}^{n}\right)$ and $i \in\{0, \ldots, n\}$.

Proof. For every $x \in \mathbb{R}^{n}$, the definition of the subdifferential implies that $\partial u_{x_{0}}(x)=\partial u\left(x-x_{0}\right)$ and therefore $\Gamma_{u_{x_{0}}}=\Gamma_{u}+\left(x_{0}, 0\right)$. Consequently,

$$
\begin{aligned}
P_{s}\left(u_{x_{0}}, \eta\right) & =\left\{x+s y:(x, y) \in \eta \cap \Gamma_{u_{x_{0}}}\right\} \\
& =\left\{x+s y:(x, y) \in \eta \cap\left(\Gamma_{u}+\left(x_{0}, 0\right)\right)\right\} \\
& =\left\{x+s y:(x, y) \in\left(\left(\eta-\left(x_{0}, 0\right)\right) \cap \Gamma_{u}\right)+\left(x_{0}, 0\right)\right\} \\
& =\left\{x+s y:(x, y) \in\left(\eta-\left(x_{0}, 0\right)\right) \cap \Gamma_{u}\right\}+x_{0} \\
& =P_{s}\left(u, \eta-\left(x_{0}, 0\right)\right)+x_{0} .
\end{aligned}
$$


Similarly, $\phi^{-1} \partial u_{\phi}(x)=\partial u\left(\phi^{-1} x\right)$ and therefore $\Gamma_{u_{\phi}}=\tilde{\phi}\left(\Gamma_{u}\right)$, which implies that

$$
\begin{aligned}
P_{s}\left(u_{\phi}, \eta\right) & =\left\{x+s y:(x, y) \in \eta \cap \Gamma_{u_{\phi}}\right\} \\
& =\left\{x+s y:(x, y) \in \eta \cap \tilde{\phi} \Gamma_{u}\right\} \\
& =\left\{x+s y:(x, y) \in \tilde{\phi}\left(\tilde{\phi}^{-1} \eta \cap \Gamma_{u}\right)\right\} \\
& =\phi\left(P_{s}\left(u, \tilde{\phi}^{-1} \eta\right)\right) .
\end{aligned}
$$

The conclusions follow from taking the Lebesgue measure of $P_{s}\left(u_{x_{0}}, \eta\right)$ and of $P_{s}\left(u_{\phi}, \eta\right)$, respectively, and from using the Steiner type formula (7.1) and the invariance of the Lebesgue measure under rotations and translations.

8.2. Hessian measures of $u$ and $u^{*}$. Let $T: \mathbb{R}^{2 n} \rightarrow \mathbb{R}^{2 n}$ be defined by $T(x, y)=(y, x)$. Given a subset $\eta$ of $\mathbb{R}^{2 n}$, we set

$$
\hat{\eta}=\left\{(x, y) \in \mathbb{R}^{n} \times \mathbb{R}^{n}:(y, x) \in \eta\right\}=T(\eta) .
$$

If $u \in \operatorname{Conv}\left(\mathbb{R}^{n}\right)$, then its conjugate function $u^{*}$ belongs to $\operatorname{Conv}\left(\mathbb{R}^{n}\right)$ as well. Moreover, by Lemma3.7, for every $(x, y) \in \mathbb{R}^{n} \times \mathbb{R}^{n}$, we have $y \in \partial u(x)$ if and only if $x \in \partial u^{*}(y)$. Hence

$$
\Gamma_{u}=T\left(\Gamma_{u^{*}}\right) .
$$

Similarly,

$$
\Gamma_{u} \cap \eta=T\left(\Gamma_{u^{*}} \cap \hat{\eta}\right)
$$

for all $\eta \in \mathcal{B}\left(\mathbb{R}^{2 n}\right)$. We easily deduce the following result, already observed in [11, Theorem 5.8].

Theorem 8.2. For $u \in \operatorname{Conv}\left(\mathbb{R}^{n}\right)$,

$$
\Theta_{i}(u, \eta)=\Theta_{n-i}\left(u^{*}, \hat{\eta}\right)
$$

for every $i=0, \ldots, n$ and $\eta \in \mathcal{B}\left(\mathbb{R}^{2 n}\right)$.

8.3. The measures $\Theta_{0}$ and $\Theta_{n}$. We consider the extremal cases $i=0$ and $i=n$ for Hessian measures.

Proposition 8.3. For every $u \in \operatorname{Conv}\left(\mathbb{R}^{n}\right)$,

$$
\Theta_{n}(u, \eta)=\mathcal{H}^{n}\left(\pi_{1}\left(\eta \cap \Gamma_{u}\right)\right), \quad \Theta_{0}(u, \eta)=\mathcal{H}^{n}\left(\pi_{2}\left(\eta \cap \Gamma_{u}\right)\right),
$$

for all $\eta \in \mathcal{B}\left(\mathbb{R}^{2 n}\right)$.

Proof. We have $P_{0}(u, \eta)=\pi_{1}\left(\eta \cap \Gamma_{u}\right)$. Hence the first equality follows from (7.1) with $s=0$. The second is obtained by applying the first one to $u^{*}$ and by Theorem 8.2 ,

A particular case of the previous result was already presented in [10, p. 3248].

\subsection{Lower dimensional domains.}

Proposition 8.4. If $u \in \operatorname{Conv}\left(\mathbb{R}^{n}\right)$, then $\Theta_{i}(u, \cdot) \equiv 0$ for every $i>\operatorname{dim}(\operatorname{dom}(u))$.

Proof. Let $\operatorname{dim}(\operatorname{dom}(u))=k$. There exists an affine subspace $E$ of $\mathbb{R}^{n}$ of dimension $k$, such that $\operatorname{dom}(u) \subset E$. Without loss of generality, we may assume that

$$
E=\left\{\left(x^{\prime}, 0\right): x^{\prime} \in \mathbb{R}^{k}\right\},
$$

where 0 stands for the zero element of $\mathbb{R}^{n-k}$. Let $F$ be the orthogonal complement of $E$, that is,

$$
F=\left\{\left(0, x^{\prime \prime}\right): x^{\prime \prime} \in \mathbb{R}^{n-k}\right\} .
$$

Define $u_{E}: \mathbb{R}^{k} \rightarrow \mathbb{R} \cup\{+\infty\}$ as the restriction of $u$ to $E$, that is, $u_{E}\left(x^{\prime}\right)=u\left(x^{\prime}, 0\right)$ for $x^{\prime} \in \mathbb{R}^{k}$. It follows from $\operatorname{dom}(u) \subset E$ that $u_{E} \in \operatorname{Conv}\left(\mathbb{R}^{k}\right)$. It is not hard to check that, for every $x^{\prime} \in \mathbb{R}^{k}$,

$$
\partial u\left(x^{\prime}, 0\right)=\left\{\left(y^{\prime}, y^{\prime \prime}\right): y^{\prime} \in \partial u_{E}\left(x^{\prime}\right), y^{\prime \prime} \in \mathbb{R}^{n-k}\right\} .
$$


Hence

$$
\Gamma_{u}=\left\{\left(\left(x^{\prime}, 0\right),\left(y^{\prime}, y^{\prime \prime}\right)\right): x^{\prime} \in \mathbb{R}^{k}, y^{\prime} \in \partial u_{E}\left(x^{\prime}\right), y^{\prime \prime} \in \mathbb{R}^{n-k}\right\} .
$$

Now let $\alpha^{\prime}, \beta^{\prime} \in \mathcal{B}\left(\mathbb{R}^{k}\right)$ and $\alpha^{\prime \prime}, \beta^{\prime \prime} \in \mathcal{B}\left(\mathbb{R}^{n-k}\right)$ with $0 \in \alpha^{\prime \prime}$. Set

$$
\eta=\alpha^{\prime} \times \alpha^{\prime \prime} \times \beta^{\prime} \times \beta^{\prime \prime} \in \mathcal{B}\left(\mathbb{R}^{2 n}\right) .
$$

For $s \geq 0$, we obtain

$$
\begin{aligned}
P_{s}(u, \eta) & =\left\{\left(x^{\prime}+s y^{\prime}, s y^{\prime \prime}\right):\left(x^{\prime}, y^{\prime}\right) \in \Gamma_{u_{E}} \cap\left(\alpha^{\prime} \times \beta^{\prime}\right), y^{\prime \prime} \in \beta^{\prime \prime}\right\} \\
& =P_{s}\left(u_{E}, \alpha^{\prime} \times \beta^{\prime}\right) \times s \beta^{\prime \prime} .
\end{aligned}
$$

Taking Lebesgue measures on both sides, we get

$$
\mathcal{H}^{n}\left(P_{s}(u, \eta)\right)=s^{n-k} \mathcal{H}^{k}\left(P_{s}\left(u_{E}, \alpha^{\prime} \times \beta^{\prime}\right)\right) .
$$

This implies that the polynomial expansion of $\mathcal{H}^{n}\left(P_{s}(u, \eta)\right)$ does not contain any term of order lower than $(n-k)$. Thus

$$
\Theta_{k+1}(u, \eta)=\cdots=\Theta_{n}(u, \eta)=0 .
$$

The conclusion now follows from the arbitrariness of $\alpha^{\prime}, \alpha^{\prime \prime}, \beta^{\prime}, \beta^{\prime \prime}$ and the non-negativity of Hessian measures.

8.5. Smooth functions. For $u \in \operatorname{Conv}\left(\mathbb{R}^{n}\right) \cap C^{2}\left(\mathbb{R}^{n}\right)$, we have

$$
\Gamma_{u}=\left\{(x, \nabla u(x)): x \in \mathbb{R}^{n}\right\},
$$

and

$$
P_{s}\left(u, \beta \times \mathbb{R}^{n}\right)=\left\{x+s \nabla u(x): x \in \beta \times \mathbb{R}^{n}\right\}
$$

for every $\beta \in \mathcal{B}\left(\mathbb{R}^{n}\right)$ and $s \geq 0$. Hence

$$
\mathcal{H}^{n}\left(P_{s}\left(u, \beta \times \mathbb{R}^{n}\right)\right)=\int_{P_{s}\left(u, \beta \times \mathbb{R}^{n}\right)} \mathrm{d} z=\int_{\beta} \operatorname{det}\left(I_{n}+s \mathrm{D}^{2} u(x)\right) \mathrm{d} x,
$$

by the change of variables $z=x+s \nabla u(x)$. On the other hand:

$$
\operatorname{det}\left(I_{n}+s \mathrm{D}^{2} u(x)\right)=\sum_{i=0}^{n}\left(\begin{array}{l}
n \\
i
\end{array}\right) s^{i}\left[\mathrm{D}^{2} u(x)\right]_{i} .
$$

By Theorem 7.1, we get

$$
\Theta_{i}\left(u, \beta \times \mathbb{R}^{n}\right)=\int_{\beta}\left[\mathrm{D}^{2} u(x)\right]_{n-i} \mathrm{~d} x
$$

for every $i \in\{0, \ldots, n\}$ and $\beta \in \mathcal{B}\left(\mathbb{R}^{n}\right)$.

\section{VAluations on CONVEX Functions}

Let $\langle\mathcal{G},+\rangle$ be an Abelian semi-group.

Definition 9.1. A function $\mathrm{Z}: \operatorname{Conv}\left(\mathbb{R}^{n}\right) \rightarrow \mathcal{G}$ is called a valuation if

$$
\mathrm{Z}(u \vee v)+\mathrm{Z}(u \wedge v)=\mathrm{Z}(u)+\mathrm{Z}(v)
$$

for every $u, v \in \operatorname{Conv}\left(\mathbb{R}^{n}\right)$ such that $u \wedge v \in \operatorname{Conv}\left(\mathbb{R}^{n}\right)$. 
We will only be interested in two cases: $\mathcal{G}=\mathbb{R}$ and $\mathcal{G}=\mathcal{M}\left(\mathbb{R}^{2 n}\right)$; the latter is the space of Borel measures on $\mathbb{R}^{2 n}$. In both situations there exists a natural choice of topology, the Euclidean topology on $\mathbb{R}$ and the topology of weak convergence on $\mathcal{M}\left(\mathbb{R}^{2 n}\right)$. A valuation $\mathrm{Z}$ is called continuous if, for every sequence $u_{k}$ in $\operatorname{Conv}\left(\mathbb{R}^{n}\right)$ epi-convergent to $u \in \operatorname{Conv}\left(\mathbb{R}^{n}\right)$, we have $\lim _{k \rightarrow+\infty} \mathrm{Z}\left(u_{k}\right)=\mathrm{Z}(u)$ in $\mathcal{G}$.

In the following definitions we use the notation introduced in the previous section for the composition of a function with a translation or a rotation. Let $\mathrm{Z}: \operatorname{Conv}\left(\mathbb{R}^{n}\right) \rightarrow \mathbb{R}$ be a valuation. We say that

(i) $\mathrm{Z}$ is translation invariant if $\mathrm{Z}\left(u_{x_{0}}\right)=\mathrm{Z}(u)$ for all $u \in \operatorname{Conv}\left(\mathbb{R}^{n}\right)$ and $x_{0} \in \mathbb{R}^{n}$;

(ii) $\mathrm{Z}$ is rotation invariant if $\mathrm{Z}\left(u_{\phi}\right)=\mathrm{Z}(u)$ for all $u \in \operatorname{Conv}\left(\mathbb{R}^{n}\right)$ and $\phi \in \mathrm{O}(n)$;

(iii) $\mathrm{Z}$ is rigid motion invariant if it is translation and rotation invariant.

(iv) $\mathrm{Z}$ is $i$-simple, for $i=1, \ldots, n$, if $\mathrm{Z}(u)=0$ whenever $u \in \operatorname{Conv}\left(\mathbb{R}^{n}\right)$ and $\operatorname{dim}(\operatorname{dom}(u)) \leq i+1$.

Clearly, all the previous definition can be repeated for functionals defined on subsets of $\operatorname{Conv}\left(\mathbb{R}^{n}\right)$.

\subsection{Measure-valued valuations.}

Theorem 9.2. For $i \in\{0, \ldots, n\}$, the function $\Theta_{i}: \operatorname{Conv}\left(\mathbb{R}^{n}\right) \rightarrow \mathcal{M}\left(\mathbb{R}^{2 n}\right)$ is a continuous and $i$-simple valuation.

Proof. Continuity and simplicity follow from Theorems 7.3 and 8.4 respectively. As for the valuation property, let $u, v \in \operatorname{Conv}\left(\mathbb{R}^{n}\right)$ be such that $u \wedge v \in \operatorname{Conv}\left(\mathbb{R}^{n}\right)$. We have to prove that

$$
\Theta_{i}(u \wedge v, \eta)+\Theta_{i}(u \vee v, \eta)=\Theta_{i}(u, \eta)+\Theta_{i}(v, \eta)
$$

for $\eta \in \mathcal{B}\left(\mathbb{R}^{2 n}\right)$. By Proposition 6.4 we have, for every $s \geq 0$,

$$
\begin{aligned}
\mathcal{H}^{n}\left(P_{s}\right. & (u \\
= & \wedge v, \eta))+\mathcal{H}^{n}\left(P_{s}(u \wedge v, \eta) \cup P_{s}(u \vee v, \eta)\right)+\mathcal{H}^{n}\left(P_{s}(u \wedge v, \eta) \cap P_{s}(u \vee v, \eta)\right) \\
= & \mathcal{H}^{n}\left(P_{s}(u, \eta) \cup P_{s}(v, \eta)\right)+\mathcal{H}^{n}\left(P_{s}(u, \eta) \cap P_{s}(v, \eta)\right) \\
= & \mathcal{H}^{n}\left(P_{s}(u, \eta)\right)+\mathcal{H}^{n}\left(P_{s}(v, \eta)\right) .
\end{aligned}
$$

The conclusion follows immediately from the Steiner type formula (7.1).

\section{Hessian Valuations}

Let $\zeta: \mathbb{R} \times \mathbb{R}^{n} \times \mathbb{R}^{n} \rightarrow \mathbb{R}$ be a continuous function. We extend its definition to include the value $+\infty$ for its first variable, setting $\zeta(+\infty, x, y)=0$ for all $(x, y) \in \mathbb{R}^{n} \times \mathbb{R}^{n}$. This extension will be always tacitly used in the rest of the paper.

We are now in a position to prove Theorem 1.1 and first establish the following result.

Theorem 10.1. Let $\zeta \in C\left(\mathbb{R} \times \mathbb{R}^{n} \times \mathbb{R}^{n}\right)$ have compact support with respect to the second and third variables. For $i \in\{0,1, \ldots, n\}$, the functional $\mathrm{Z}_{\zeta, i}$ : $\operatorname{Conv}\left(\mathbb{R}^{n}\right) \rightarrow \mathbb{R}$, defined by

$$
\mathrm{Z}_{\zeta, i}(u)=\int_{\Gamma_{u}} \zeta(u(x), x, y) \mathrm{d} \Theta_{i}(u,(x, y)),
$$

is an i-simple and continuous valuation on $\operatorname{Conv}\left(\mathbb{R}^{n}\right)$.

Remark. If $u \in \operatorname{Conv}\left(\mathbb{R}^{n}\right) \cap C^{2}\left(\mathbb{R}^{n}\right)$, then, by (8.1) and (8.2),

$$
\left.\mathrm{Z}_{\zeta, i}(u)=\int_{\Gamma_{u}} \zeta(u(x), x, y) \mathrm{d} \Theta_{i}(u,(x, y))=\int_{\mathbb{R}^{n}} \zeta(u(x), x, \nabla u(x))\left[\mathrm{D}^{2} u(x)\right)\right]_{n-i} \mathrm{~d} x .
$$

Hence, all statements of Theorem 1.1 follow from the above theorem. 
The proof of Theorem 10.1 requires some preparatory steps.

Lemma 10.2. Let $\zeta \in C\left(\mathbb{R} \times \mathbb{R}^{n} \times \mathbb{R}^{n}\right)$ and $u \in \operatorname{Conv}\left(\mathbb{R}^{n}\right)$. The function $\xi: \Gamma_{u} \rightarrow \mathbb{R}$, defined as $\xi(x, y)=\zeta(u(x), x, y)$, is continuous on $\Gamma_{u}$.

Proof. For $r>0$, by Proposition 4.1, the function $u_{r}=\operatorname{reg}_{r} u$ has $\operatorname{dom}\left(u_{r}\right)=\mathbb{R}^{n}$ and $u(x)=u_{r}(x)$ for $x$ such that $|y| \leq 1 / r$ for every $y \in \partial u(x)$. Hence

$$
\xi(x, y)=\zeta(u(x), x, y)=\zeta\left(u_{r}(x), x, y\right)
$$

for $(x, y) \in \Gamma_{u}^{r}$. As $u_{r}$ is continuous in $\mathbb{R}^{n}$, $\xi$ is continuous on $\Gamma_{u}^{r}$. As $r>0$ is arbitrary, the conclusion follows.

Proposition 10.3. Let $\zeta \in C\left(\mathbb{R} \times \mathbb{R}^{n} \times \mathbb{R}^{n}\right)$ have compact support with respect to the second and third variables. For every $u \in \operatorname{Conv}\left(\mathbb{R}^{n}\right)$, the function $\xi: \Gamma_{u} \rightarrow \mathbb{R}$, defined as $\xi(x, y)=\zeta(u(x), x, y)$, is summable with respect to the measure $\Theta_{i}(u, \cdot)$ for $i \in\{0, \ldots, n\}$.

Proof. We recall that the support of $\Theta_{i}(u, \cdot)$ is contained in $\Gamma_{u}$. Set $\zeta_{+}=\max \{\zeta, 0\}$ and $\zeta_{-}=\min \{\zeta, 0\}$. We clearly have

$$
\xi_{+}(x, y):=\max \{\xi(x, y), 0\}=\zeta_{+}(u(x), x, y), \quad \xi_{-}(x, y):=\min \{\xi(x, y), 0\}=\zeta_{-}(u(x), x, y) .
$$

By Lemma 10.2, the function $\xi_{+}$is continuous and hence integrable with respect to the Borel measure $\Theta_{i}(u, \cdot)$ over $\Gamma_{u}$. Let us prove that $\xi_{+}$is in fact summable (the proof for $\xi_{-}$is completely analogous). As $\zeta$ has compact support in $(x, y)$, there exists $r>0$ such that $\zeta(t, x, y)=0$ whenever $|x| \geq r$ or $|y| \geq r$. Moreover, there exists $u_{r} \in \operatorname{Conv}\left(\mathbb{R}^{n}\right)$ such that $\operatorname{dom}\left(u_{r}\right)=\mathbb{R}^{n}$, and $u(x)=u_{r}(x)$ for all $x \in \pi_{1}\left(\Gamma_{u}^{r}\right)$. Hence

$$
\xi(x, y)=\zeta\left(u_{r}(x), x, y\right)
$$

for all $(x, y) \in \Gamma_{u}$. By continuity, $u_{r}$ is bounded in the set $\left\{x \in \mathbb{R}^{n}:|x| \leq r\right\}$ and there exists a constant $c>0$ such that

$$
\xi_{+}(x, y) \leq c
$$

for all $(x, y) \in \Gamma_{u}$. Consequently,

$$
\int_{\mathbb{R}^{2 n}} \zeta\left(u_{r}(x), x, y\right) \mathrm{d} \Theta_{i}\left(u_{r},(x, y)\right) \leq c \Theta_{i}\left(u_{r}, \eta\right)
$$

for $\eta=\left\{(x, y) \in \mathbb{R}^{2 n}:|x|,|y| \leq r\right\}$. The conclusion follows from the fact that $\Theta_{i}\left(u_{r}, \cdot\right)$ is locally finite (see $(7.2)$ ).

10.1. Proof of Theorem 10.1, By Proposition 10.3, the integral (10.1) is finite.

For simplicity, for an arbitrary $u \in \operatorname{Conv}\left(\mathbb{R}^{n}\right)$ we write $\Theta_{i}(u, \cdot)$ as $\Theta(u, \cdot)$. As remarked above, the support of $\Theta(u, \cdot)$ is contained in $\Gamma_{u}$. Moreover, by Theorem 7.5, we have

$$
\int_{\Gamma_{u}} \zeta(u(x), x, y) \mathrm{d} \Theta(u,(x, y))=\int_{\Gamma_{u}} \zeta(u(x), x, y) \theta(u,(x, y)) \mathrm{d} \mathcal{H}^{n}(x, y),
$$

for a suitable non-negative, $\mathcal{H}^{n}$-measurable function $\theta(u, \cdot)$ defined on $\Gamma_{u}$. Let $r>0$ be such that $\zeta(t, x, y)=0$ if either $|x|>r$ or $|y|>r$. For $u \in \operatorname{Conv}\left(\mathbb{R}^{n}\right)$, we set $u_{r}=\operatorname{reg}_{r} u$ and use the properties established in Proposition 6.5 and Proposition 7.6 .

1. The valuation property. Let $u, v \in \operatorname{Conv}\left(\mathbb{R}^{n}\right)$ be such that $u \wedge v \in \operatorname{Conv}\left(\mathbb{R}^{n}\right)$. Set

$$
\Sigma=\Gamma_{u} \cup \Gamma_{v}=\Gamma_{u \vee v} \cup \Gamma_{u \wedge v}
$$

(see Proposition 6.3). We will consider a generic point $(x, y) \in \Sigma$ such that the densities

$$
\theta(u,(x, y)), \theta(v,(x, y)), \theta(u \vee v,(x, y)), \theta(u \wedge v,(x, y))
$$


are well defined; this happens to be true for $\mathcal{H}^{n}$-a.e. $(x, y) \in \Sigma$. In view of (10.2), it is enough to prove that for $\mathcal{H}^{n}$-a.e. $(x, y) \in \Sigma$, we have

$$
\begin{aligned}
& \zeta(u(x), x, y) \theta(u,(x, y))+\zeta(v(x), x, y) \theta(v,(x, y)) \\
& =\zeta((u \vee v)(x), x, y) \theta(u \vee v,(x, y))+\zeta((u \wedge v)(x), x, y) \theta(u \wedge v,(x, y))
\end{aligned}
$$

Indeed, integrating over $\Sigma$ we get

$$
\mathrm{Z}_{\zeta, i}(u)+\mathrm{Z}_{\zeta, i}(v)=\mathrm{Z}_{\zeta, i}(u \vee v)+\mathrm{Z}_{\zeta, i}(u \wedge v) .
$$

It will suffice to prove (10.3) under the assumption $|y| \leq r$, as it is trivially true for $|y|>r$ since $\zeta$ vanishes at each point where it is computed.

By the valuation property of Hessian measures (Theorem 9.2), we deduce

$$
\theta(u \vee v,(x, y))+\theta(u \wedge v,(x, y))=\theta(u,(x, y))+\theta(v,(x, y)) \quad \text { for } \mathcal{H}^{n} \text {-a.e. }(x, y) \in \Sigma \text {. }
$$

This shows in particular that (10.3) holds true for every $(x, y)$ such that $u(x)=v(x)$. Next assume that $\left(x_{0}, y_{0}\right)$ is such that $u\left(x_{0}\right) \neq v\left(x_{0}\right)$ and without loss of generality, assume that $u\left(x_{0}\right)>v\left(x_{0}\right)$.

Case 1: Let $\left(x_{0}, y_{0}\right) \notin \Gamma_{u} \cap \Gamma_{v}$. Then we also have, by Proposition 6.3, $\left(x_{0}, y_{0}\right) \notin \Gamma_{u \vee v} \cap \Gamma_{u \wedge v}$. Assume that $\left(x_{0}, y_{0}\right) \in \Gamma_{u} \backslash \Gamma_{v}$. From (5.1), $y_{0} \in \partial u\left(x_{0}\right)=\partial(u \vee v)\left(x_{0}\right)$; therefore

$$
\left(x_{0}, y_{0}\right) \in \Gamma_{u \vee v} \backslash \Gamma_{u \wedge v} .
$$

Hence

$$
\theta\left(v,\left(x_{0}, y_{0}\right)\right)=\theta\left(u \wedge v,\left(x_{0}, y_{0}\right)\right)=0
$$

In this case (10.4) reduces to

$$
\theta\left(u \vee v,\left(x_{0}, y_{0}\right)\right)=\theta\left(u,\left(x_{0}, y_{0}\right)\right)
$$

and (10.3) follows from multiplying both sides of the previous equation by $(u \vee v)\left(x_{0}\right)=u\left(x_{0}\right)$. The case $\left(x_{0}, y_{0}\right) \in \Gamma_{v} \backslash \Gamma_{u}$ is completely analogous.

Case 2: Let $\left(x_{0}, y_{0}\right) \in \Gamma_{u} \cap \Gamma_{v}$. Then $u_{r}\left(x_{0}\right)=u\left(x_{0}\right)>v\left(x_{0}\right)=v_{r}\left(x_{0}\right)$. Let $U$ be a neighborhood of $x_{0}$ such that $u_{r}>v_{r}$ in $U$ (which exists by the continuity of $u_{r}$ and $v_{r}$ ). Hence

$$
u_{r} \wedge v_{r} \equiv v_{r}, \quad u_{r} \vee v_{r} \equiv u_{r} \quad \text { in } U \text {. }
$$

Then we also have $\partial\left(u_{r} \wedge v_{r}\right)(x)=\partial v_{r}(x)$ and $\partial\left(u_{r} \vee v_{r}\right)(x)=\partial v_{r}(x)$ for every $x \in U$, and this implies that

$$
P_{s}\left(u_{r} \wedge v_{r}, \eta\right)=P_{s}\left(v_{r}, \eta\right), \quad P_{s}\left(u_{r} \vee v_{r}, \eta\right)=P_{s}\left(u_{r}, \eta\right)
$$

for every $s \geq 0$ and for every $\eta \subset \mathbb{R}^{n} \times \mathbb{R}^{n}$ such that $\pi_{1}(\eta) \subset U$. Consequently,

$$
\Theta\left(u_{r} \wedge v_{r}, \eta\right)=\Theta\left(v_{r}, \eta\right), \quad \Theta\left(u_{r} \vee v_{r}, \eta\right)=\Theta\left(u_{r}, \eta\right)
$$

for every such $\eta$. We deduce that

$$
\theta\left(u_{r} \wedge v_{r}, x_{0}\right)=\theta\left(v_{r}, x_{0}\right), \quad \theta\left(u_{r} \vee v_{r}, x_{0}\right)=\theta\left(u_{r}, x_{0}\right) .
$$

By Proposition 4.3 and Proposition 7.6, we have

$$
\theta\left(u \wedge v, x_{0}\right)=\theta\left(v, x_{0}\right), \quad \theta\left(u \vee v, x_{0}\right)=\theta\left(u, x_{0}\right) .
$$

These equations, combined with the obvious relations

$$
(u \wedge v)\left(x_{0}\right)=v\left(x_{0}\right), \quad(u \vee v)\left(x_{0}\right)=u\left(x_{0}\right)
$$

lead to (10.3). 
2. Continuity. Let $u_{k}$ be a sequence in $\operatorname{Conv}\left(\mathbb{R}^{n}\right)$ epi-converging to $u \in \operatorname{Conv}\left(\mathbb{R}^{n}\right)$. For simplicity, we set $w_{k}=\left(u_{k}\right)_{r}=\operatorname{reg}_{r} u_{k}$ and $w=u_{r} \operatorname{reg}_{r} u$.

$$
\begin{aligned}
\left|\mathrm{Z}_{\zeta, i}\left(u_{k}\right)-\mathrm{Z}_{\zeta, i}(u)\right| & =\left|\int_{\mathbb{R}^{2 n}} \zeta\left(u_{k}(x), x, y\right) \mathrm{d} \Theta\left(u_{k},(x, y)\right)-\int_{\mathbb{R}^{2 n}} \zeta(u(x), x, y) \mathrm{d} \Theta(u,(x, y))\right| \\
& =\left|\int_{\mathbb{R}^{2 n}} \zeta\left(w_{k}(x), x, y\right) \mathrm{d} \Theta\left(u_{k},(x, y)\right)-\int_{\mathbb{R}^{2 n}} \zeta(w(x), x, y) \mathrm{d} \Theta(u,(x, y))\right| \\
& \leq \int_{\mathbb{R}^{2 n}}\left|\zeta\left(w_{k}(x), x, y\right)-\zeta(w(x), x, y)\right| \mathrm{d} \Theta\left(u_{k},(x, y)\right)+ \\
& +\left|\int_{\mathbb{R}^{2 n}} \zeta(w(x), x, y) \mathrm{d} \Theta\left(u_{k},(x, y)\right)-\int_{\mathbb{R}^{2 n}} \zeta(w(x), x, y) \mathrm{d} \Theta(u,(x, y))\right| .
\end{aligned}
$$

By Proposition 5.5, the sequence $w_{k}$ epi-converges to $w$; as $w_{k}$ and $w$ are finite in $\mathbb{R}^{n}$, the convergence is uniform on compact sets. As $\zeta$ is continuous and with compact support with respect to $x$ and $y$, the sequence $\zeta\left(w_{k}(x), x, y\right)$ converges uniformly to $\zeta(w(x), x, y)$ in $\mathbb{R}^{2 n}$ as $k \rightarrow+\infty$. Hence for an arbitrary $\varepsilon>0$, and for sufficiently large $k$, we have

$$
\int_{\mathbb{R}^{2 n}}\left|\zeta\left(w_{k}(x), x, y\right)-\zeta(w(x), x, y)\right| \mathrm{d} \Theta\left(u_{k},(x, y)\right) \leq \varepsilon \Theta\left(u_{k}, C\right)
$$

where $C$ is a compact subset of $\mathbb{R}^{2 n}$ such that $\zeta(t, x, y)=0$ if $(x, y) \notin C$. On the other hand, as Hessian measures are locally finite, there exists a constant $c>0$ depending only on $C$ such that

$$
\Theta\left(u_{k}, C\right) \leq c
$$

for every $k \in \mathbb{N}$. This proves that

$$
\lim _{k \rightarrow+\infty} \int_{\mathbb{R}^{2 n}}\left|\zeta\left(w_{k}(x), x, y\right)-\zeta(w(x), x, y)\right| \mathrm{d} \Theta\left(u_{k},(x, y)\right)=0 .
$$

Set $\xi(x, y)=\zeta(w(x), x, y)$ for $(x, y) \in \mathbb{R}^{n}$. Recall that $w$ is a convex function such that $\operatorname{dom}(w)=$ $\mathbb{R}^{n}$; in particular it is continuous in $\mathbb{R}^{n}$, so that $\xi$ is continuous. Moreover, $\xi$ has compact support, as $\zeta$ has this property with respect to $(x, y)$. As the sequence of measures $\Theta\left(u_{k}, \cdot\right)$ converges weakly to $\Theta(u, \cdot)$, we have

$$
\lim _{k \rightarrow+\infty} \int_{\mathbb{R}^{2 n}} \zeta(w(x), x, y) \mathrm{d} \Theta\left(u_{k},(x, y)\right)-\int_{\mathbb{R}^{2 n}} \zeta(w(x), x, y) \mathrm{d} \Theta(u,(x, y))=0,
$$

which proves the weak continuity.

3. Simplicity. Proposition 8.4 implies simplicity.

\subsection{Invariance properties.}

Proposition 10.4. Let $\zeta \in C\left(\mathbb{R} \times \mathbb{R}^{n} \times \mathbb{R}^{n}\right)$ have compact support with respect to the second and third variables. If there exists $\xi \in C\left(\mathbb{R} \times[0,+\infty)^{2}\right)$ such that $\zeta(t, x, y)=\xi(t,|x|,|y|)$ on $\mathbb{R} \times \mathbb{R}^{n} \times \mathbb{R}^{n}$, then the valuation $\mathrm{Z}_{\zeta, i}$ defined by (10.1) is rotation invariant.

Proof. Let $\phi \in \mathrm{O}(n), u \in \operatorname{Conv}\left(\mathbb{R}^{n}\right)$ and $u_{\phi}$ be defined as in Section 8 . We have

$$
\mathrm{Z}_{\zeta, i}\left(u_{\phi}\right)=\int_{\Gamma_{u_{\phi}}} \xi\left(u_{\phi}(x),|x|,|y|\right) \mathrm{d} \Theta_{i}\left(u_{\phi},(x, y)\right) .
$$


As observed in the proof of Proposition 8.1, we have $\Gamma_{u_{\phi}}=\tilde{\phi} \Gamma_{u}$. The conclusion follows by the change of variables

and Proposition 8.1

$$
(\bar{x}, \bar{y})=(\phi x, \phi y)=\tilde{\phi}(x, y)
$$

10.3. Composing a valuation with the conjugate function. Let $\mathcal{G}$ be a topological Abelian semigroup and $\mathrm{Z}: \operatorname{Conv}\left(\mathbb{R}^{n}\right) \rightarrow \mathcal{G}$ a continuous valuation. By Proposition 3.4 and Proposition 3.5, the functional $\mathrm{Z}^{*}: \operatorname{Conv}\left(\mathbb{R}^{n}\right) \rightarrow \mathcal{G}$ defined by

$$
\mathrm{Z}^{*}(u)=\mathrm{Z}\left(u^{*}\right)
$$

is a continuous valuation as well.

Assume that $\mathcal{G}=\mathbb{R}$ and that $\mathrm{Z}=\mathrm{Z}_{\zeta, i}$ is of the form (10.1). Then for every $u \in \operatorname{Conv}\left(\mathbb{R}^{n}\right)$,

$$
\begin{aligned}
\mathrm{Z}_{\zeta, i}^{*}(u) & =\int_{\mathbb{R}^{2 n}} \zeta\left(u^{*}(x), x, y\right) \mathrm{d} \Theta_{i}\left(u^{*},(x, y)\right) \\
& =\int_{\mathbb{R}^{2 n}} \zeta(\langle x, y\rangle-u(y), x, y) \mathrm{d} \Theta_{i}\left(u^{*},(x, y)\right) \\
& =\int_{\mathbb{R}^{2 n}} \zeta(\langle x, y\rangle-u(x), y, x) \mathrm{d} \Theta_{n-i}(u,(y, x)) \\
& =\mathrm{Z}_{\bar{\zeta}, n-i}(u),
\end{aligned}
$$

where we have used (iii) of Lemma 3.7 and Theorem 8.2, and we set

$$
\bar{\zeta}(t, x, y)=\zeta(\langle x, y\rangle-t, y, x)
$$

on $\mathbb{R} \times \mathbb{R}^{n} \times \mathbb{R}^{n}$. This shows in particular that $\mathrm{Z}^{*}$ is still a Hessian valuation of the form (10.1).

10.4. The cases $i=0$ and $i=n$. Let $\mathrm{Z}$ be of the form (10.1), with $i=n$ :

$$
\mathrm{Z}_{\zeta, n}(u)=\int_{\Gamma_{u}} \zeta(u(x), x, y) \mathrm{d} \Theta_{n}(u,(x, y)),
$$

for a suitable $\zeta \in C\left(\mathbb{R} \times \mathbb{R}^{n} \times \mathbb{R}^{n}\right)$, having compact support with respect to $x$ and $y$.

Let $u \in \operatorname{Conv}\left(\mathbb{R}^{n}\right)$; if $\operatorname{dim}(\operatorname{dom}(u))<n$, then $\mathrm{Z}_{\zeta, n}(u)=0$ by the simplicity property stated in Theorem 10.1. Assume that $\operatorname{dim}(\operatorname{dom}(u))=n$, and let

$$
D=\{x \in \operatorname{int}(\operatorname{dom}(u)): u \text { is differentiable at } x\} .
$$

As $u$ is differentiable $\mathcal{H}^{n}$-a.e. on $\operatorname{dom}(u)$, we have

$$
\mathcal{H}^{n}(\operatorname{dom}(u) \backslash D)=0 .
$$

Hence, setting

$$
\Gamma_{u, D}=\left\{(x, y) \in \Gamma_{u}: x \in D\right\}
$$

by Proposition 8.3 ,

$$
\begin{aligned}
\mathrm{Z}_{\zeta, n}(u) & =\int_{\Gamma_{u, D}} \zeta(x, u(x), \nabla u(x)) \mathrm{d} \Theta_{n}(u,(x, y)) \\
& =\int_{D} \zeta(x, u(x), \nabla u(x)) \mathrm{d} x
\end{aligned}
$$

We conclude that

$$
\mathrm{Z}_{\zeta, n}(u)=\int_{\operatorname{dom}(u)} \zeta(x, u(x), \nabla u(x)) \mathrm{d} x
$$


for $u \in \operatorname{Conv}\left(\mathbb{R}^{n}\right)$. Using this and Theorem 8.2 we also get

$$
\mathrm{Z}_{\zeta, 0}(u)=\int_{\operatorname{dom}\left(u^{*}\right)} \zeta\left(\left\langle\nabla u^{*}(y), y\right\rangle-u^{*}(y), \nabla u^{*}(y), y\right) \mathrm{d} y
$$

for $u \in \operatorname{Conv}\left(\mathbb{R}^{n}\right)$.

\section{Valuations on Convex and Coercive Functions}

We can now prove Theorem 1.2 and first establish the following result.

Theorem 11.1. Let $\zeta \in C\left(\mathbb{R} \times \mathbb{R}^{n}\right)$ have compact support and let $i \in\{0, \ldots, n\}$. The functional $\mathrm{Z}_{\zeta, i}: \operatorname{Conv}_{\text {coe }}\left(\mathbb{R}^{n}\right) \rightarrow \mathbb{R}$, defined by

$$
\mathrm{Z}_{\zeta, i}(u)=\int_{\mathbb{R}^{2 n}} \zeta(u(x), y) \mathrm{d} \Theta_{i}(u,(x, y)),
$$

is a continuous, translation invariant, $i$-simple valuation. Moreover, if there exists $\zeta \in C(\mathbb{R} \times[0,+\infty))$ such that $\zeta(t, y)=\xi(t,|y|)$ on $\mathbb{R} \times \mathbb{R}^{n}$, then $\mathrm{Z}_{\zeta, i}$ is also rotation invariant.

Proof. As $\zeta$ has compact support and $u$ is coercive, the function $(x, y) \mapsto \zeta(u(x), y)$ has compact support as well. By Lemma 10.2 this function is also continuous on $\Gamma_{u}$; hence, as Hessian measures are locally finite, the integral in (11.1) is always finite. The proofs of the valuation property, continuity and rotation invariance are the same as in the case described in the previous section.

In order to prove translation invariance, let $u \in \operatorname{Conv}_{\text {coe }}\left(\mathbb{R}^{n}\right)$ and $x_{0} \in \mathbb{R}^{n}$. For the translated function $u_{x_{0}}$, we have

$$
\mathrm{Z}_{\zeta, i}\left(u_{x_{0}}\right)=\int_{\mathbb{R}^{2 n}} \zeta\left(u\left(x-x_{0}\right), y\right) \mathrm{d} \Theta_{i}\left(u_{x_{0}},(x, y)\right) .
$$

The claim follows by the change of variables $\left(x-x_{0}, y\right)=(\bar{x}, \bar{y})$ and Proposition 8.1 .

Remark. If $\zeta$ is as in the previous theorem and $u \in \operatorname{Conv}_{\text {coe }}\left(\mathbb{R}^{n}\right) \cap C^{2}\left(\mathbb{R}^{n}\right)$, then

$$
\mathrm{Z}_{\zeta, i}(u)=\int_{\mathbb{R}^{n}} \zeta(u(x), \nabla u(x))\left[\mathrm{D}^{2} u(x)\right]_{n-i} \mathrm{~d} x .
$$

Hence Theorem 11.1 implies Theorem 1.2.

Remark. We have seen that a sufficient condition on $\zeta \in C\left(\mathbb{R} \times \mathbb{R}^{n}\right)$ such that for $i \in\{0, \ldots, n\}$, the integral

$$
\mathrm{Z}_{\zeta, i}(u)=\int_{\mathbb{R}^{2 n}} \zeta(u(x), y) \mathrm{d} \Theta_{i}(u,(x, y))
$$

is finite for every $u \in \operatorname{Conv}_{\text {coe }}\left(\mathbb{R}^{n}\right)$, is that $\zeta$ has compact support. On the other hand, in some special cases necessary and sufficient conditions for integrability are known. For instance, in [15] is proved that if $i=n$ and $\zeta=\zeta(t)$ is non-negative, then (11.2) is finite for every $u \in \operatorname{Conv}_{\operatorname{coe}}\left(\mathbb{R}^{n}\right)$ if and only if

$$
\int_{0}^{+\infty} t^{n-1} \zeta(t) \mathrm{d} t<+\infty
$$

It would be interesting to understand what the corresponding conditions are in the general case. In other words, it would be interesting to have for given $i$ an explicit description of the space of functions

$$
\operatorname{Int}_{i}\left(\mathbb{R} \times \mathbb{R}^{n}\right)=\left\{\zeta \in C\left(\mathbb{R} \times \mathbb{R}^{n}\right): \int_{\mathbb{R}^{2 n}} \zeta(u(x), y) \mathrm{d} \Theta_{i}(u,(x, y))<\infty \text { for every } u \in \operatorname{Conv}_{\operatorname{coe}}\left(\mathbb{R}^{n}\right)\right\}
$$

For the one-dimensional case, some progress in this direction was obtained in [32]. 
11.1. Comparison with level-set based valuations. Let $u \in \operatorname{Conv}_{\mathrm{coe}}\left(\mathbb{R}^{n}\right)$. For $t \in \mathbb{R}$, we consider the sublevel set $\{u \leq t\}=\left\{x \in \mathbb{R}^{n}: u(x) \leq t\right\}$. By the semi-continuity, convexity and coercivity of $u$, this is either a compact convex subset of $\mathbb{R}^{n}$, that is, a convex body, or the empty set. In particular, for every $k \in\{0, \ldots, n\}$, the $k$ th quermassintegral $W_{k}(\{u \leq t\})$ is well defined (with the convention $W_{k}(\emptyset)=0$ for every $k$ ).

Let $\omega \in C(\mathbb{R})$ have compact support. For $k \in\{0, \ldots, n\}$, functionals of the form

$$
\mathrm{X}_{k}(u)=\int_{\mathbb{R}} \omega(t) W_{k}(\{u \leq t\}) \mathrm{d} t
$$

have been considered on $\operatorname{Conv}_{\text {coe }}\left(\mathbb{R}^{n}\right)$ in [7] (with slightly different assumptions on $\omega$ ), and in the context of log-concave or quasi-concave functions in [5, 12, 28, 29].

The functional in (11.3) defines a continuous, rigid motion invariant valuation on $\operatorname{Conv}_{\text {coe }}\left(\mathbb{R}^{n}\right)$. Indeed, the assumption of compact support on $\omega$ and coercivity assure that it is finite. The valuation property was observed in [7], and rigid motion invariance is an immediate consequence of the rigid motion invariance of quermassintegrals. Continuity with respect to epi-convergence can be proved using Lemma 5 in [15], which concerns the relation between epi-convergence and convergence of level sets. We refer to functionals of the form (11.3) as to level-set based valuations.

When $k=n$, we have $W_{n}(K)=\kappa_{n}$ (the volume of the unit ball in $\mathbb{R}^{n}$ ) for every (non-empty) convex body. Hence

$$
\mathrm{X}_{n}(u)=\xi\left(\min _{\mathbb{R}^{n}} u\right)
$$

for $u \in \operatorname{Conv}_{\text {coe }}\left(\mathbb{R}^{n}\right)$, where, for $t \in \mathbb{R}$, we set

$$
\xi(t)=\kappa_{n} \int_{t}^{\infty} \omega(s) \mathrm{d} s .
$$

For $k=0$, as $W_{0}$ is the Lebesgue measure, by the Layer Cake Principle (see Section 6.3 in [7]), we have the equivalent representation

$$
\mathrm{X}_{0}(u)=\int_{\operatorname{dom}(u)} \xi(u(x)) \mathrm{d} x
$$

for $u \in \operatorname{Conv}_{\text {coe }}\left(\mathbb{R}^{n}\right)$, where, for $t \in \mathbb{R}$, we now set

$$
\xi(t)=\int_{t}^{+\infty} \omega(s) \mathrm{d} s .
$$

In particular, (11.4) is a valuation which can be written as a Hessian valuation and as a level-set based valuation.

The following examples show that the family of Hessian valuations is not included in that of level-set based valuations.

Example 11.2. Let $n=1$. Note that there are only two types of level-set based valuations on $\operatorname{Conv}_{\text {coe }}(\mathbb{R})$ :

$$
\mathrm{X}_{0}(u)=\xi_{0}\left(\min _{\mathbb{R}} u\right), \quad \mathrm{X}_{1}(u)=\int_{\operatorname{dom}(u)} \xi_{1}(u(x)) \mathrm{d} x,
$$

where $\xi_{0}, \xi_{1} \in C(\mathbb{R})$. Let $\zeta \in C\left(\mathbb{R}^{2}\right)$ be even with respect to second variable and have compact support. We consider the Hessian valuation Z defined on $\operatorname{Conv}_{\text {coe }}\left(\mathbb{R}^{n}\right)$ as

$$
\mathrm{Z}(u)=\int_{\Gamma_{u}} \zeta(u(x), y) \mathrm{d} \Theta_{0}(u,(x, y)) .
$$


Assume that $\mathrm{Z}$ can be written as linear combination of level-set based valuations, that is,

$$
\mathrm{Z}(u)=\xi_{0}(\min (u))+\int_{\operatorname{dom}(u)} \xi_{1}(u(x)) \mathrm{d} x
$$

for suitable $\xi_{0}$ and $\xi_{1}$. Evaluating (11.5) on functions of the form $u(x)=t+I_{[-r, r]}(x)$ with $t \in \mathbb{R}$ and $r>0$ and using Proposition 8.3 , we obtain

$$
\int_{\mathbb{R}} \zeta(t, y) \mathrm{d} y=\xi_{0}(t)+2 r \xi_{1}(t)
$$

As the last term is the only one depending on $r$, we get $\xi_{1} \equiv 0$. Hence (11.5) becomes

$$
\mathrm{Z}(u)=\xi_{0}\left(\min _{\mathbb{R}} u\right) .
$$

Plugging the function $u(x)=t+s|x|$ into the last equality with $t \in \mathbb{R}$ and $s>0$, we deduce

$$
\int_{-s}^{s} \zeta(t, y) \mathrm{d} y=2 \int_{0}^{s} \zeta(t, y) \mathrm{d} y=\xi_{0}(t)
$$

for $t \in \mathbb{R}$ and $s>0$. Thus we obtain $\zeta \equiv 0$.

Example 11.3. Let $\zeta=\zeta(t, s)$ be a function in $C(\mathbb{R} \times[0, \infty))$ of compact support. Consider the Hessian valuation $\mathrm{Z}$ defined on $\operatorname{Conv}_{\text {coe }}\left(\mathbb{R}^{n}\right)$ by

$$
\mathrm{Z}(u)=\int_{\Gamma_{u}} \zeta(u(x),|y|) \mathrm{d} \Theta_{n}(u,(x, y))=\int_{\operatorname{dom}(u)} \zeta(u(x),|\nabla u(x)|) \mathrm{d} x .
$$

Assume that $\mathrm{Z}$ can be written as linear combination of level-set based valuations

$$
\mathrm{Z}(u)=\sum_{k=0}^{n} \mathrm{X}_{k}(u)
$$

Note that $\mathrm{Z}$ is $n$-simple, i.e., it vanishes for all $u$ such that $\operatorname{dim}(\operatorname{dom}(u))<n$. The only level-set based valuations with this property are those for $k=0$. It is not hard to see that this implies that the right hand-side of the previous inequality reduces to the term $\mathrm{X}_{0}$, hence, on $\operatorname{Conv}_{\text {coe }}\left(\mathbb{R}^{n}\right)$,

$$
\mathrm{Z}(u)=\int_{\operatorname{dom}(u)} \xi(u(x)) \mathrm{d} x
$$

for some function $\xi \in C(\mathbb{R})$. We evaluate (11.6) at functions $u$ of the form $u(x)=s|x|+I_{B_{r}^{n}}$, with $s, r>0$, obtaining

$$
\int_{0}^{r} \zeta(s t, s) t^{n-1} \mathrm{~d} t=\int_{0}^{r} \xi(s t) t^{n-1} \mathrm{~d} t .
$$

As $r>0$ is arbitrary, we deduce $\zeta(t, s)=\xi(t)$ for all $t \in \mathbb{R}$ and $s>0$, which is possible only if $\xi$ does not depend on the second variable.

\section{The Space of 1-homogeneous Convex Functions}

We analyze connections of the results on valuations on convex functions and valuations on convex bodies. Let

$$
\operatorname{Conv}_{\text {hom }}\left(\mathbb{R}^{n}\right)=\left\{u \in \operatorname{Conv}\left(\mathbb{R}^{n}\right): \operatorname{dom}(u)=\mathbb{R}^{n}, u \text { is } 1 \text {-homogeneous }\right\} .
$$

It is well known that $u \in \operatorname{Conv}_{\text {hom }}\left(\mathbb{R}^{n}\right)$ if and only if there exists a convex body $K$ in $\mathbb{R}^{n}$ such that $u=h_{K}$, the support function of $K$ (see Example 3.2). We recall that $\mathcal{K}^{n}$ denotes the family of convex bodies in $\mathbb{R}^{n}$. 
We want to discuss valuations on $\operatorname{Conv}_{\text {hom }}\left(\mathbb{R}^{n}\right)$ and first look at Hessian measures on this space. Denote by $u$ a generic element of $\operatorname{Conv}_{\text {hom }}\left(\mathbb{R}^{n}\right)$ and by $K$ the convex body such that $u=h_{K}$. For a point $y \in \operatorname{bd}(K)$, let $N(K, y)$ be the normal cone to $K$ at $y$. For $x \neq 0$, we have $y \in \partial u(x)$ if and only if $y \in \operatorname{bd}(K)$ and $x \in N(K, y)$; moreover, $\partial u(0)=K$ (see [35, Theorem 1.7.4]). Hence

$$
\Gamma_{u}=(\{0\} \times K) \cup\left\{(x, y) \in \mathbb{R}^{2 n}: y \in \operatorname{bd}(K), x \in N(K, y)\right\} .
$$

In particular,

$$
\Gamma_{u} \subset \mathbb{R}^{n} \times K
$$

The 1-homogeneity of $u$ implies that the subdifferential is 0 -homogeneous, that is, $\partial u(t x)=\partial u(x)$ for $x \in \mathbb{R}^{n}$ and $t>0$. Hence, for every $\eta \subset \mathbb{R}^{2 n}, s \geq 0$ and $t>0$,

$$
\begin{aligned}
P_{s}(u, t \eta) & =\{t x+s y: x \in \eta, y \in \partial u(x)\} \\
& =t\left\{x+\frac{s}{t} y:(x, y) \in \eta \cap \Gamma_{u}\right\} \\
& =t P_{s / t}(u, \eta) .
\end{aligned}
$$

Taking Lebesgue measures and using the Steiner formula (7.1), we obtain the following homogeneity property for Hessian measures of support functions,

$$
\Theta_{i}(u, t \eta)=t^{i} \Theta_{i}(u, \eta)
$$

for $\eta \in \mathcal{B}\left(\mathbb{R}^{2 n}\right)$ and $i \in\{0, \ldots, n\}$.

Hessian measures of support functions are closely connected to support measures of the corresponding convex bodies. For the definition of the support measures $\Theta_{0}(K, \cdot), \ldots, \Theta_{n}(K, \cdot)$ of a convex body $K$, we refer to [35, Theorem 4.2.1]. The following relation was established in [11, Corollary 5.9]. For every $\alpha \in \mathcal{B}\left(\mathbb{S}^{n-1}\right), \beta \in \mathcal{B}\left(\mathbb{R}^{n}\right)$ and $i \in\{1, \ldots, n\}$ :

$$
\Theta_{i}(u, \hat{\alpha} \times \beta)=\frac{1}{n} \Theta_{n-i}(K, \beta \times \alpha),
$$

where $\hat{\alpha}$ is the convex hull $\alpha \cup\{0\}$. In particular,

$$
\Theta_{i}\left(u, B^{n} \times \mathbb{R}^{n}\right)=\Theta_{i}\left(u, \Gamma_{u} \cap\left(B^{n} \times \mathbb{R}^{n}\right)\right)=\frac{1}{n} \Theta_{n-i}\left(K, \mathbb{R}^{n} \times \mathbb{S}^{n-1}\right)=W_{n-i}(K)
$$

where $W_{j}(K)$, for $j \in\{0, \ldots, n\}$, is the $j$ th quermassintegral of $K$. The previous equality extends to the case $i=0$ as

$$
\Theta_{0}\left(u, B^{n} \times \mathbb{R}^{n}\right)=\Theta_{0}\left(u, \Gamma_{u} \cap\left(B^{n} \times \mathbb{R}^{n}\right)\right)=\mathcal{H}^{n}\left(\pi_{2}\left(\Gamma_{u}\right)\right)=\mathcal{H}^{n}(K)=W_{0}(K) .
$$

A real-valued valuation on $\mathcal{K}^{n}$ is a functional $\mathrm{Y}: \mathcal{K}^{n} \rightarrow \mathbb{R}$ which has the additivity property

$$
\mathrm{Y}(K \cup L)+\mathrm{Y}(K \cap L)=\mathrm{Y}(K)+\mathrm{Y}(L)
$$

for every $K, L \in \mathcal{K}^{n}$ such that $K \cup L \in \mathcal{K}^{n}$. The valuation $\mathrm{Y}$ is continuous, if it is continuous with respect to the Hausdorff metric.

Hadwiger's celebrated classification theorem is the following result (see [19]).

Theorem 12.1 (Hadwiger). A functional $\mathrm{Y}: \mathcal{K}^{n} \rightarrow \mathbb{R}$ is a continuous valuation, invariant under rotations and translations if and only if there exist $c_{0}, \ldots, c_{n} \in \mathbb{R}$ such that

$$
\mathrm{Y}(K)=\sum_{i=0}^{n} c_{i} W_{i}(K)
$$

for every $K \in \mathcal{K}^{n}$. 
To rewrite Hadwiger's theorem as a result on $\operatorname{Conv}_{\text {hom }}\left(\mathbb{R}^{n}\right)$, we need the following facts. We associate with $\mathrm{Y}: \mathcal{K}^{n} \rightarrow \mathbb{R}$ the functional $\overline{\mathrm{Y}}: \operatorname{Conv}_{\text {hom }}\left(\mathbb{R}^{n}\right) \rightarrow \mathbb{R}$, defined as $\overline{\mathrm{Y}}\left(h_{K}\right)=\mathrm{Y}(K)$. First, let $K_{j}$ be a sequence of convex bodies in $\mathbb{R}^{n}$ and let $u_{j}=h_{K_{j}}$ for every $j \in \mathbb{N}$. The sequence $K_{j}$ converges to a convex body $K$ with respect to the Hausdorff metric if and only if the sequence of functions $u_{j}$ converges pointwise to $u=h_{K}$ in $\mathbb{R}^{n}$ (and uniformly on compact sets). This is in turn equivalent to the fact that $u_{j}$ epi-converges to $u$. Hence, $\mathrm{Y}: \mathcal{K}^{n} \rightarrow \mathbb{R}$ is continuous with respect to the Hausdorff metric if and only if $\bar{Y}: \operatorname{Conv}_{\text {hom }}\left(\mathbb{R}^{n}\right) \rightarrow \mathbb{R}$ is epi-continuous.

Second, if $K, L \in \mathcal{K}^{n}$ are such that $K \cup L \in \mathcal{K}^{n}$, then

$$
h_{K \cup L}=h_{K} \vee h_{L}, \quad h_{K \cap L}=h_{K} \wedge h_{L} .
$$

This follows from Example 3.2 and Proposition 3.4. Hence, if $Y: \mathcal{K}^{n} \rightarrow \mathbb{R}$ is a valuation, then $\overline{\mathrm{Y}}: \operatorname{Conv}_{\text {hom }}\left(\mathbb{R}^{n}\right) \rightarrow \mathbb{R}$ is also a valuation.

Third, for every $\phi \in \mathrm{O}(n)$, the support function of $\phi K$ is given by $h_{\phi K}(x)=h_{K}\left(\phi^{t} x\right)$ for every $x \in \mathbb{R}^{n}$. Hence, $\mathrm{Y}: \mathcal{K}^{n} \rightarrow \mathbb{R}$ is rotation invariant if and only if $\overline{\mathrm{Y}}$ is also rotation invariant.

Finally, for every $x_{0} \in \mathbb{R}^{n}$, the support function of the convex body $K+x_{0}$ is given by

$$
h_{K+x_{0}}(x)=h_{K}(x)+\left\langle x, x_{0}\right\rangle=u(x)+\left\langle x, x_{0}\right\rangle
$$

for every $x \in \mathbb{R}^{n}$. Hence, $\mathrm{Y}: \mathcal{K}^{n} \rightarrow \mathbb{R}$ is translation invariant if and only if $\overline{\mathrm{Y}}(u+w)=\overline{\mathrm{Y}}(w)$ for every linear function $w: \mathbb{R}^{n} \rightarrow \mathbb{R}$. In general, we say that $\mathrm{Z}: \operatorname{Conv}_{\text {hom }}\left(\mathbb{R}^{n}\right) \rightarrow \mathbb{R}$ is unchanged by addition of linear functions if $\mathrm{Z}(u+w)=\mathrm{Z}(u)$ for every $u \in \operatorname{Conv}_{\text {hom }}\left(\mathbb{R}^{n}\right)$ and linear function $w$.

As a consequence of the previous considerations, we obtain the following version of Hadwiger's theorem.

Theorem 12.2 (Hadwiger). A functional $\mathrm{Z}: \operatorname{Conv}_{\text {hom }}\left(\mathbb{R}^{n}\right) \rightarrow \mathbb{R}$ is a continuous and rotation invariant valuation that is unchanged by addition of linear functions if and only if there exists $c_{0}, \ldots, c_{n} \in \mathbb{R}$ such that

$$
\mathrm{Z}(u)=\sum_{i=0}^{n} c_{i} \Theta_{i}\left(u, B^{n} \times \mathbb{R}^{n}\right)
$$

for every $u \in \operatorname{Conv}_{\mathrm{hom}}\left(\mathbb{R}^{n}\right)$.

Without requiring that the functionals are unchanged by addition of linear functions, we obtain many further functionals.

Theorem 12.3. Let $\zeta \in C(\mathbb{R} \times[0,+\infty))$. For $i \in\{0, \ldots, n\}$, the functional $\mathrm{Z}_{\zeta, i}: \operatorname{Conv}_{\mathrm{hom}}\left(\mathbb{R}^{n}\right) \rightarrow \mathbb{R}$, defined by

$$
\mathrm{Z}_{\zeta, i}(u)=\int_{B^{n} \times \mathbb{R}^{n}} \zeta(u(x),|y|) \mathrm{d} \Theta_{i}(u,(x, y)),
$$

is a continuous and rotation invariant valuation on $\operatorname{Conv}_{\mathrm{hom}}\left(\mathbb{R}^{n}\right)$. Consequently, $K \mapsto \mathrm{Z}_{\zeta, i}\left(h_{K}\right)$ is a continuous and rotation invariant valuation on $\mathcal{K}^{n}$.

Proof. Finiteness of $\mathrm{Z}_{\zeta, i}$ follows from the compactness of $\Gamma_{u} \cap\left(B^{n} \times \mathbb{R}^{n}\right)$, the continuity of $u$ and $\zeta$, and the local finiteness of Hessian measures. The proofs of the valuation property, continuity and rotation invariance are as in the proofs of Theorem 10.1

Alesker [1] obtained a classification of continuous and rotation invariant valuations on convex bodies that are polynomial with respect to translations and showed that these are functionals of type (12.1). 
Acknowledgments. The work of Monika Ludwig and Fabian Mussnig was supported, in part, by Austrian Science Fund (FWF) Project P25515-N25. The work of Monika Ludwig was also supported by the National Science Foundation under Grant No. DMS-1440140 while she was in residence at the Mathematical Sciences Research Institute in Berkeley, California, during the Fall 2017 semester. The work of Andrea Colesanti was supported by the G.N.A.M.P.A. and by the F.I.R. project 2013: Geometrical and Qualitative Aspects of PDE's.

\section{REFERENCES}

[1] S. Alesker, Continuous rotation invariant valuations on convex sets, Ann. Math. 149 (1999), 977-1005.

[2] S. Alesker, Valuations on convex sets, non-commutative determinants, and pluripotential theory, J. Geom. Anal. 18 (2008), 651-686.

[3] S. Alesker, Valuations on convex functions and convex sets and Monge-Ampère operators, preprint, arXiv:1703.08778 (2017).

[4] Y. Baryshnikov, R. Ghrist, and M. Wright, Hadwiger's Theorem for definable functions, Adv. Math. 245 (2013), 573586.

[5] S. G. Bobkov, A. Colesanti, and I. Fragalà, Quermassintegrals of quasi-concave functions and generalized PrékopaLeindler inequalities, Manuscripta Math. 143 (2014), 131-169.

[6] L. Caffarelli, L. Nirenberg and J. Spruck, The Dirichlet problem for nonlinear second order elliptic equations, III: functions of the eigenvalues of the Hessian, Acta Math. 155 (1985), 261-301.

[7] L. Cavallina and A. Colesanti, Monotone valuations on the space of convex functions, Anal. Geom. Metr. Spaces 3 (2015), 167-211.

[8] A. Colesanti, A Steiner type formula for convex functions, Mathematika 44 (1997), 195-214.

[9] A. Colesanti and I. Fragalà, The first variation of the total mass of log-concave functions and related inequalities, Adv. Math. 244 (2013), 708-749.

[10] A. Colesanti, D. Hug, Steiner type formulas and weighted measures of singularities for semi-convex functions, Trans. Amer. Math. Soc. 352 (2000), 3239-3263.

[11] A. Colesanti, D. Hug, Hessian measures of semi-convex functions and applications to support measures of convex bodies, Manuscripta Math. 101 (2000), 209-238.

[12] A. Colesanti and N. Lombardi, Valuations on the space of quasi-concave functions, Geometric aspects of functional analysis, 71-105, Lecture Notes in Mathematics 2169, Springer International Publishing, Cham, 2017.

[13] A. Colesanti, N. Lombardi, L. Parapatits, Translation invariant valuations on the space of quasi-concave functions, preprint, arXiv: 1703.06867(2017).

[14] A. Colesanti, M. Ludwig, F. Mussnig, Minkowski valuations on convex functions, Calc. Var. Partial Differential Equations 56 (2017), 56:162.

[15] A. Colesanti, M. Ludwig, F. Mussnig, Valuations on convex functions, Int. Math. Res. Not. IMRN, in press.

[16] A. Colesanti, P. Salani, Generalised solutions of Hessian equations, Bull. Austral. Math. Soc. 56 (1997), 459-466.

[17] H. Federer, Geometric Measure Theory, Springer, Berlin, 1979.

[18] J. H. G. Fu, Monge-Ampère functions. I, Indiana Univ. Math. J. 38 (1989), 745-771.

[19] H. Hadwiger, Vorlesungen über Inhalt. Oberfläche und Isoperimetrie, Springer, Berlin, 1957.

[20] J.-B. Hiriart-Urruty, Lipschitz r-continuity of the approximate subdifferential of a convex function, Math. Scand. 47 (1980), 123-134.

[21] J.-B. Hiriart-Urruty, Extensions of Lipschitz functions, J. Math. Anal. Appl. 77 (1980), 539-554.

[22] H. Kone, Valuations on Orlicz spaces and L ${ }^{\varphi}$-star sets, Adv. in Appl. Math. 52 (2014), 82-98.

[23] M. Ludwig, Fisher information and valuations, Adv. Math. 226 (2011), 2700-2711.

[24] M. Ludwig, Valuations on function spaces, Adv. Geom. 11 (2011), 745-756.

[25] M. Ludwig, Valuations on Sobolev spaces, Amer. J. Math. 134 (2012), 827-842.

[26] M. Ludwig, Covariance matrices and valuations, Adv. in Appl. Math. 51 (2013), 359-366.

[27] D. Ma, Real-valued valuations on Sobolev spaces, Sci. China Math. 59 (2016), 921-934.

[28] V. Milman, L. Rotem, Mixed integrals and related inequalities, J. Funct. Analysis 264 (2013), 570-604.

[29] V. Milman, L. Rotem, $\alpha$-concave functions and a functional extension of mixed volumes, Electr. Res. Announcements Math. Sci. 20 (2013), 1-11.

[30] F. Mussnig, Valuations on log-concave functions, preprint, arXiv:1707.06428(2017).

[31] M. Ober, $L_{p}$-Minkowski valuations on $L^{q}$-spaces, J. Math. Anal. Appl. 414 (2014), 68-87. 
[32] G. Paoli, Condizioni di integrabilità per integrali hessiani in dimensione uno, Tesi di Laurea Magistrale, Università di Firenze (in preparation).

[33] T. Rockafellar, Convex Analysis, Princeton University Press, Princeton, 1970.

[34] T. Rockafellar, R. Wets, Variational Analysis, Springer, Berlin, 1998.

[35] R. Schneider, Convex Bodies: the Brunn-Minkowski Theory, Second expanded ed., Encyclopedia of Mathematics and its Applications, vol. 151, Cambridge University Press, Cambridge, 2014.

[36] N. S. Trudinger and X.-J. Wang, Hessian measures. II, Ann. of Math. (2) 150 (1999), 579-604.

[37] A. Tsang, Valuations on $L^{p}$ spaces, Int. Math. Res. Not. 20 (2010), 3993-4023.

[38] A. Tsang, Minkowski valuations on $L^{p}$-spaces, Trans. Amer. Math. Soc. 364 (2012), 6159-6186.

[39] T. Wang, Semi-valuations on BV $\left(\mathbb{R}^{n}\right)$, Indiana Univ. Math. J. 63 (2014), 1447-1465.

Andrea Colesanti: Dipartimento di Matematica e Informatica “U. Dini” Università degli Studi Di FirenZe, Viale Morgagni 67/A - 50134, FirenZe, ITALY

E-mail address: colesant@math.unifi.it

Monika Ludwig: Institut für Diskrete Mathematik und Geometrie, Technische Universität Wien, WIEDNER HAUPTSTRASSE 8-10/1046, 1040 WIEN, AUSTRIA

E-mail address: monika.ludwig@tuwien.ac.at

Fabian Mussnig: Institut fÜR Diskrete Mathematik und Geometrie, Technische Universität Wien, WIEDNER HAUPTSTRASSE 8-10/1046, 1040 WIEN, AUSTRIA

E-mail address: fabian.mussnigealumni.tuwien.ac.at 